\title{
A tool for designing tree-like concentration gradient generators for
}

\section{lab-on-a-chip applications ${ }^{\dagger}$}

\author{
Milad Ebadi $^{\text {a }}$, Khashayar Moshksayan ${ }^{\text {a\#}}$, Navid Kashaninejad ${ }^{\text {b\# }}$, Mohammad Said Saidi ${ }^{*}$, Nam-Trung \\ Nguyen $^{\text {b* }}$ \\ ${ }^{a}$ Department of Mechanical Engineering, Sharif University of Technology, Tehran 11155, Iran \\ ${ }^{\mathrm{b}}$ Queensland Micro- and Nanotechnology Centre, Griffith University, Nathan Campus, 170 Kessels \\ Road, Brisbane, QLD 4111, Australia \\ \# These authors contributed equally to this work* Correspondence: mssaidi@sharif.edu; nam- \\ trung.nguyen@griffith.edu.au \\ $\dagger$ Electronic supplementary information (ESI) available.
}

\begin{abstract}
Concentration gradient generators (CGGs) help biologists to perform large scale, fast and highthroughput experiments. This paper introduces a design tool called Tree-like Concentration gradient generator Design Tool (TCDT). The performance of this tool is validated both numerically and experimentally. Three CGGs were fabricated using three different fabrication methods and design parameters. The performance of these devices was examined using the measurement of fluorescent and dye intensity. The performance of the design tool for non-linear and multi-drug concentration gradient generations was investigated as well. In addition, a method was developed to investigate the multi-drug concentration effect, which can be the starting point for off-the-shelf usage of the CGGs in personalized medicine. Experimental data, as well as numerical results, confirmed that the output concentrations of the CGGs were within five percent of the values predicted by the design tool. This open-source software is available online for public use.
\end{abstract}

Keywords: Tree-like concentration gradient generator; open-source CGG; fluorescence measurement; microfluidics; CFD 


\section{Introduction}

In most biosynthesis activities, concentration of chemicals significantly affects cellular behavior $(\underline{\mathrm{Hu}}$ et al., 2017). The transfer of gases from cells to the environment is completely dependent on the concentration gradient of the gases in the environment and within the tissue (Truskey et al., 2004). Various phenomena in the body such as cell division and cellular movement (Chung et al., 2005; Huang et al., 2017; Lee et al., 2011; Park et al., 2007), immune function (Joanne Wang et al., 2008; Kothapalli et al., 2011), wound healing (Wang, 2009), tumorigenesis (Chiang et al., 2012), and osteogenesis ( the tissue. In cancer medications, an optimized concentration of a drug should be used for patients to achieve the best outcome. Therefore, for each patient, different concentrations of the drug should be tested first, and the best dose needs to be applied later (Ruppen et al., 2015). For this reason, different methods have been developed to generate concentration gradients.

Concentration gradient generators (CGGs) are among the most efficient devices employed to precisely generate various concentration gradients. In this context, concentration gradients refer to the different output concentrations of the device with a predictable trend. Advances in microtechnology, particularly in microfluidics, enhances the design and function of CGGs significantly. These enhancements have been generally attributed to the reduction of the dimensions of the device. Methods for generating concentration gradients in CCGs can be grouped in four categories: membrane methods (Boyden, 1962; Zicha et al., 1991; Zigmond SH, 1973), micro pipetting (Gundersen and Barrett, 1979), serial dilution (integra_support, 2017), and microfluidic methods (Abhyankar et al., 2006; Bui et al., 2011; Huang et al., 2017; Kilinc et al., 2016; Nguyen et al., 2016). 
Microfluidic CGGs have emerged as essential components in most cell culture and organ-ona-chip platforms (Kashaninejad et al., 2016; Moshksayan et al., 2018a; Moshksayan et al., 2018b). Microfluidic CGGs divide into subgroups such as the diffusion-based method (Abhyankar et al., 2006), pressure balance (Kilinc et al., 2016), T-junction (Bui et al., 2011), hydrogels (guyen et al., 2016), and tree-like CGGs (TCGGs) (Huang et al., 2017).

TCGGs are frequently used for drug screening in microfluidic cell culture platforms because they can generate more precise and stable concentrations (Chang et al., 2014b; Diao et al., 2006; DiCicco and Neethirajan, 2014; Hong et al., 2016; Huang et al., 2017; Jang et al., 2011; Jeon et al., 2000; Keenan and Folch, 2008; Kothapalli et al., 2011; Lee et al., 2010; Nguyen et al., 2016; $\underline{\text { Park et }}$ al., 2007; Tretkoff, 2005; Ye et al., 2007). Moreover, this subgroup is easier to use and more robust against variations in environmental conditions (Jeon et al., 2000). One of the first attempts to design TCGGs was reported by Jeon et al. (Jeon et al., 2000). The authors designed a TCGG based on an analytical model of concentration and pressure. The flow rate was $50 \mu 1 / \mathrm{min}$, and the dimension of the channel was $50 \mu \mathrm{m}$, both in width and height. The generated concentration was precise and stable near the design flow rate. However, the output concentration deviated from the predicted values at higher flow rate. This research was a reference for most of the subsequent studies on CGGs (Huang et al., 2017; Jeon et al., 2002; Jin et al., 2016; Lim and Park, 2018; Lin et al., 2004; Walker et al.., $\underline{2005) .}$

TCGGs can generate non-linear concentration gradients using different methods. However, the most common method is using different inlet flow rates because it is cheaper and easier than other methods (Toh et al., 2014). Lin et al. designed multiple tree-like CGGs with multiple inlets to generate nonlinear concentration gradients (Lin et al., 2004). They generated logarithmic and exponential profiles using different designs. There are some other methods to design non-linear concentration gradients like electroosmosis method (Glawdel et al., 2009; Jain et al., 2010). Their other application in electrokinetics, other than fluid motion, is the preconcentration of charged particles. This method has 
several applications in biochemical and environmental industries. Nevertheless, channel clogging is a major challenge associated with such method, and several attempts have been made to prevent the issue of blocking. For instance, Harrison et al. presented a method for low conductivity medium for preconcentration of polystyrene particles and E.coli cells (Harrison et al., 2015). Ren et al. studied effective electrokinetic enrichment of fluorescent polystyrene nanoparticles on ideally polarizable metal strips using hybrid electroosmotic kinetics as bi-phase induced-charge electroosmosis (BICEO) actuated in a four-terminal spiral electrode array (Ren et al., 2019). Liu et al. developed a reliable method for flexible regulation on the spatial-temporal evolution of iondepletion layer by electroconvective mixing using microscopic physical description of electrothermal method (Liu et al., 2019).

Another application of TCGGs is generating multi-drug concentration gradients. Liu et al. designed a multilayer CGG using the monolithic fabrication method (Liu et al., 2008). Their device could generate various combinations such that three drugs were mixed equally or each pair of the drugs existed with the same concentration. Lee et al. designed another CGG using rapid prototyping method that could generate multiple concentration gradients of one or more species based on the number of layers of the chip (Lee et al., 2010). The fabrication of both devices was complicated and expensive. Another creative design using multi-drugs in the experiment was performed by Yang et al. (Yang et al., 2011). The authors introduced a circular design in the orientation of the output channels to generate a single layer chip for cell apoptosis and multi-drug drug screening.

Designing TCGGs is a complicated procedure and needs in-depth knowledge of mass transport and fluid dynamics. Thus, most researchers prefer to adopt their designs from previously reported devices to bypass the tedious procedure (Chang et al., 2014a; DiCicco and Neethirajan, 2014; Huang et al., 2017; Jin et al., 2016; Toh et al., 2014). In a previous work (Rismanian et al., 2019), we introduced a dimensionless number, which is constant for each type of CGG. This number is useful for designing mixers but cannot model the entire CGG because it does not consider the connecting channels and the different flow rates. Depending on the flow rate and the material used in 
the device design, design parameters such as channel width, mixers' length, culture media, and cells are unique for each application. Therefore, this study uses the analytical model of concentration, and a simple procedure was developed to facilitate the design of CGGs with minimum knowledge of the physics of CGGs and engineering principles.

This paper reports a novel Tree-like Concentration gradient generator Design Tool (TCDT) based on the fluid mechanics' models and previous research on CGGs. By using this tool, researchers can customize TCGGs based on the required flow rate, fabrication method, material, and dimensions. The validity of the tool was evaluated using both computational fluid dynamics (CFD) simulations and experimental investigation for three different TCGGs, each of which was fabricated by a different method. Furthermore, we report a method for examination of multi-drug screening based on Yang's design of CGGs (Yang et al., 2011). Yang's approach with slight modifications could serve as ofthe-shelf usage of the CGGs for personalized medicine (Mark et al., 2012). The method finds the best concentration of the drug for each person, reducing side-effects as well as increasing the effectiveness of the drug (Ruppen et al., 2015). The verifications of the output concentrations were based on the Rhodamine-B diffusion coefficients. TCDT executable file has been uploaded on the following link (https://github.com/Biofluidmechtools/TCDT.git) for public use, and the source code is available on demand.

\section{Materials and methods}

2.1 The architecture of the tree-like concentration gradient generator design tool

The Tree-like Concentration gradient generator Design Tool (TCDT) uses the corrected mathematical model that will be explained in the upcoming sections to estimate the device performance. The required information for the user is the fabrication method, the required flow rate, diffusion coefficient, dimension constraints, maximum shear stress on the wall, and other fabrication and 
material constraints that the user provides as input for the design (Fig. 1A). The detailed information needed to design the device and the manual of the tool are reported in the ESI.

\subsection{Mathematical model}

TCGGs produce the concentration gradient in the presence of flow. Because at low velocities and small dimensions, the Reynolds number is on the order of unity leading to laminar flow in most cases (Keenan and Folch, 2008). In a low-Reynolds-number laminar flow, mixing occurs through diffusion and convective mass transfer (Shames, 2003). Therefore, the channels should be sufficiently long to facilitate proper diffusive mixing. (Cengel, 2010). The mathematical model is based on the analytical solution of the flow and the analytical diffusion model of two parallel flows in a rectangular channel.

\subsection{Fluidic-electric analogy}

CGGs are made from some similar blocks of mixers that have the same fluid dynamic properties. Each mixer could be modeled as a resistor and the CGG as an electric circuit. Using this method (Eq. (1)), the flow is analogous to electric current, and the pressure drop is analogous to electric voltage difference (Eq. (2)). With this method, the resistance and the flow rate in each mixer can be found (Nguyen, 2012a).

$$
\begin{gathered}
\Delta P=R_{f} Q \\
R_{f}=3 \mu L\left(a^{3} b\left(1-\frac{192 a}{\pi^{5} b} \sum_{n=1}^{\infty} \frac{\tanh \left((2 n-1) \frac{b \pi}{2 a}\right)}{(2 n-1)^{5}}\right)\right)^{-1}
\end{gathered}
$$

In Eq. (1), $R_{\mathrm{f}}$ is the channel resistance and, $\Delta P$ and $Q$ are the pressure drop and the flow rate of the channel, respectively. In Eq. (2), $\mu$ is the fluid viscosity, $L$ is the length of the channel, and $a$ is the width of the rectangular channel. 
One of the capabilities of the TCGGs is generating non-linear concentration gradients by introducing different flow rate ratios in each inlet (Fig. 1B). The flow rate ratio for the desired shape of the output concentration can be calculated using the above fluidic-electric analogy. The flow rate of each mixer (i.e., curved channels that bend continuously and is the part where the mixing effect takes place, Fig. 1B) can be calculated using the pressure drop over each mixing channel. The output concentrations can be calculated using the inlet flow rates and concentrations of each mixer. Hence, by having the flow rate of each mixer and the input concentration by using the conservation of mass, the output concentration is calculated from the first stage (i.e., each level that the substance dilutes, Fig. 1B) to the last stage of the device. The mixers, which are located between the same interconnecting lines (i.e., channels that connect the mixers, Fig. 1B), have the same pressure drop. For linear gradients, all mixers in one stage have the same fraction of the fluid flow of each inlet. Therefore, the flow of each mixing channel could be measured by dividing the total flow rate to the number of the mixers in each stage, and each outlet (Fig. 1B) concentration is the linear interpolation between the inlet concentrations. For the devices that have nonlinear output, the flow of each mixer should be calculated using the fluidic-electric analogy (Jeon et al., 2000).

\subsection{Concentration model}

As discussed in the previous section, the fluidic-electric analogy can only determine the discharge rate, which provides the output concentration under the assumption of complete mixing. This method fails to provide any information about the quality of mixing between the two substances. To address this limitation, the length and width of each stage of the device should be appropriately designed to facilitate complete mixing. Accordingly, a new model for the diffusion of two substances in the

device's channels was provided. The diffusion of the two low concentration solutions follows the mass transfer equation (Eq. (3)) (Crank, 1979). 


$$
\nabla \cdot(\vec{u} C)=D \nabla^{2} C
$$

In Eq. (3), $u$ is the velocity of the fluid, $C$ is the concentration of the species and $D$ is the diffusion coefficient of the species. The significant part of the diffusion between the two solutions occurs in the mixing channels. Therefore, the diffusion between the two parallel flows in the channels was simulated before designing the mixers. First, the Péclet number $(\mathrm{Pe})[P e=u l / D$, where $l$ is the characteristic length of the CGG (width of the channel)] should be calculated to determine the dominant mixing mechanism between convection and diffusion. For concentration gradient applications, the highest velocity in a microchannel is about $u=10 \mathrm{~cm} / \mathrm{s}$ (Nguyen, 2012b). The mass diffusion coefficient for Rhodamine-B in a culture medium is $D=4 \times 10^{-1} \mathrm{~m}^{2} / \mathrm{s}$ (Toh et al., 2014). The characteristic length for the micro-channels of CGGs, $l$, is 50 to $100 \mu \mathrm{m}$ (Gao et al., 2012; Hong et al., 2016; Huang et al., 2017; Jeon et al., 2000; Jin et al., 2016; Lin et al., 2004; Walker et al., 2005). With the above typical values, the Pe number, with respect to longitudinal velocity and diffusion normal to velocity, is between 1,250 to 25,000 . Because of the high Pe, the convection term in the equation is significant. However, we need to consider the following assumptions to solve the equation. First, by assuming a high aspect ratio microchannel $(\mathrm{H}>>\mathrm{W}$, where $\mathrm{H}$ and $\mathrm{W}$ are height and width of the channel, respectively), the diffusion terms become negligible along the $y$ and $\mathrm{z}$ directions, where $y$ and $\mathrm{z}$ are the positions along the height and the length of the mixer, respectively. Second, we assume a constant and fully developed one-directional velocity profile in the channel. Accordingly, by applying these assumptions, the solution of the concentration model becomes as follows (Crank, 1979).

$$
C(z, x)=C_{0}\left\{\frac{1}{2}+\frac{2}{\pi} \sum_{n=1}^{\infty} \frac{1}{n} \sin \left(\frac{n \pi}{2}\right) \cos \left(\frac{n \pi x}{W}\right) \exp \left(\frac{-D n^{2} \pi^{2} z}{\bar{u} W^{2}}\right)\right\}
$$


In Eq. (4), $C$ is the concentration, $C_{0}$ is the inlet concentration, $x$ is the position along the width of the mixer, $D$ is the diffusion coefficient, $\bar{u}$ is the average flow velocity, and $W$ is the width of the channel.

To investigate the validity of the assumption of the fully developed flow, we need to consider Dean number (De). It is the ratio of the centrifugal forces to inertial forces and for conventional channels; it is the product of Reynolds number (Re) and square root of the curvature of the channel (Eq. 5) (Nguyen, 2012a).

$D e=\operatorname{Re} \sqrt{\frac{a}{R}}$

where a is the hydraulic diameter of the channel, and $\mathrm{R}$ is the radius of the curvature in the channel. For Dean number less than 40, the effect of the curvature is negligible (Ligrani, 1994). Nevertheless, for conventional CGG designs, the curvature radius is in the order of a millimeter, and the width of the channels is in the order of tenth of millimeters. The Reynolds number is in the order of one. Thus, the Dean number is far below 40, and the stream will stay fully developed in all the length of the channel because the inertial forces are dominant over the centrifugal forces and their effects. It should be noted that these assumptions become invalid when the height and the width of the channel are at the same order of magnitude, i.e., for $\mathrm{H} \approx \mathrm{W}$. In that case, one must optimize the governing equations and the models using CFD optimizations.

\subsection{CFD model}

The 3D model of the device was generated in a CAD tool. The device was modeled using a customized CFD code for validating and improving the mathematical model to generate the improved mathematical model based on optimization techniques (Rao, 1996) to decrease the effects of assumptions mentioned in Section 2.4. The model is based on laminar flow with diluted species in high-aspect-ratio channels. The solvent was water, and the solute was the desired material with a 
diffusion coefficient between $5 \times 10^{-9}$ and $5 \times 10^{-11} \mathrm{~m}^{2} / \mathrm{s}$ (Gendron et al., 2008; Kendig, 2003). The channels were $50 \mu \mathrm{m}$ in height and $200 \mu \mathrm{m}$ in width. To certify that the solution was correct, grid independency analysis was performed.

\subsection{Soft lithography}

The devices were fabricated by standard polydimethylsiloxane (PDMS) soft lithography from the fabricated molds. The molds were washed using deionized (DI) water and isopropanol before soft lithography to remove all the dust. For the positive molds (3D-printed and micromachined molds), PDMS pre-polymer and the curing agent (Silgard 184, Dow Corning, Midland, MI, USA) were mixed in the $\mathrm{W} / \mathrm{W}$ ratio of 10:1 and poured onto the molds followed by degassing in a vacuum chamber. The mixture was then crosslinked in an oven at $70{ }^{\circ} \mathrm{C}$ for $4 \mathrm{~h}$. Subsequently, the PDMS substrate was peeled off the mold, its edges were cut, and the inlets and outlets were punched using a 1-mm biopsy puncher.

For the negative molds fabricated with the photolithography method, PDMS double molding was utilized according to the method developed by Kwapiszewska et al. (Kwapiszewska et al., 2016). Briefly, after going through the above procedure, the PDMS substrate was kept in the oven at $100{ }^{\circ} \mathrm{C}$ for 48 hours for thermal aging (Kwapiszewska et al., 2016). Then, the PDMS substrate was used as a positive mold to fabricate the final PDMS device. The final PDMS parts were then bonded to glass substrates. Prior to plasma bonding, the glass substrates were washed with water and detergent and then with DI water and isopropanol.

\subsection{Mold fabrication method}

We used micromachining, 3D printing, and photolithography for making the mold. Micro milling was used for the fabrication of a positive mold on a Poly(methyl methacrylate) (PMMA) using a 
computer numerical control (CNC) milling machine (Seiko CNC Inc., Tokyo, Japan). A 0.4 mm CNC cutting tool was used to fabricate the device mold.

For 3D printing, a stereolithography printer Form2 (Formlabs Inc, Somerville, Massachusetts, United States) was used to fabricate the positive mold. The device has a minimum feature size of 150 $\mu \mathrm{m}$. In order to make the design compatible with the fabrication method, the distance between the channels was chosen to be larger to prevent the fluid within the channels from leaking into each other.

For photolithography, a negative mold was patterned on a silicon wafer using SU8-2050 negative photoresist (MicroChem Co., Newton, MA, USA). Briefly, $4 \mathrm{ml}$ of the photoresist was poured on a 4-inch silicon wafer followed by spin coating by Spin Coater ET-SC-V1 (SATEECO Inc., Tehran, Iran) according to the manufacturer's protocol to achieve a $50-\mu \mathrm{m}$ thick photoresist film. Then, the soft bake was carried out at $65^{\circ} \mathrm{C}$ and $95^{\circ} \mathrm{C}$ for 3 minutes and 9 minutes, respectively. Subsequently, the photoresist was placed under a UV exposure with a dose of $160 \mathrm{~mJ} / \mathrm{cm}^{2}$ using a mask aligner ET-MA-V1 (SATEECO Inc., Tehran, Iran) and a plastic mask. The coated wafer was then placed on a hot plate for the post-exposure bake $\left(2\right.$ minutes of $65^{\circ} \mathrm{C}$ and 10 minutes of $\left.95{ }^{\circ} \mathrm{C}\right)$ and was developed.

\subsection{Concentration measurement}

In order to measure the output concentration of the devices, two image processing-based methods were used, namely, the fluorescence intensity measurement and food dye intensity measurement. The images were taken by an MD-50, CMOS camera (Micro-shot Technology Limited, Guangzhou, China), using an LCM400 inverted microscope and florescent module (Labomed.Inc, Los Angeles, USA). The flow was pumped into the device using an EH-003 syringe pump (SATEECO Inc, Tehran, Iran). 1-ml insulin syringes and $2 \mathrm{~mm}$ Teflon tubes were used for flow injection at the inlet. 


\subsubsection{Fluorescence intensity measurement}

The fluorescence emission intensity measurement was done using Rhodamine-B (Fig. 1C) and the fluorescence intensity was processed using the Python software (Python, 2019). To quantify the intensity of the fluorescent light for each output concentration in each test, a grayscale image algorithm was developed, in which each image is a light intensity matrix of image pixels (Girod et al., 1999). For each output concentration, three outputs were taken with a time interval of 5 minutes. Finally, the results of each experiment were processed by evaluating the median value of the grayscale intensity of each photo and the median value of the three images. To overcome the dark noise in the images, their intensity matrices were normalized to the intensity of the highest and lowest intensity of the fluorescence substance in each experiment. In order to validate the results, the desired concentrations were generated manually and measured using the same equipment and algorithms. In order to show the effectiveness of the measurement technique, the predicted concentration in each case was manually generated using conventional method of mixing by a micropipette. The mixture was then injected into the device, and the photos were taken under the same conditions of the experiments. Subsequently, the results of those measurements are labeled as "hand made" in Figs 4F, 5A, and 8D."

\subsubsection{Food dye intensity measurement}

To measure the concentration using food dye intensity measurement method, red, blue, and yellow food dyes (TEIF Food Inc., Tehran, Iran) were introduced into the chip, and the images were taken from the outlets of each of the device. A customized code was generated in Python to determine the concentration of the output fluid based on the RGB (red, green, blue), Grayscale and HSV (hue, saturation, value) methods. In the HSV method, the image is a matrix whose arrays contain three 
values indicating the hue, saturation, and value (Girod et al., 1999). This method of representing the color matrix helps measure the color regardless of the light intensity on the sample (Girod et al., 1999).

The hue of the output was determined using the color image matrix. Subsequently, by normalizing color image matrices to the highest and the lowest hue in each experiment branch, the color of each output was identified. Using this method, the dark noise was minimized in the image. Another advantage of this method is improving the quality of the results by omitting the effect of imaging angles and brightness (Ramesan et al., 2016). Similarly, for this method also the "hand made" concentration was generated in order to examine the accuracy of the measurement technique. 
A

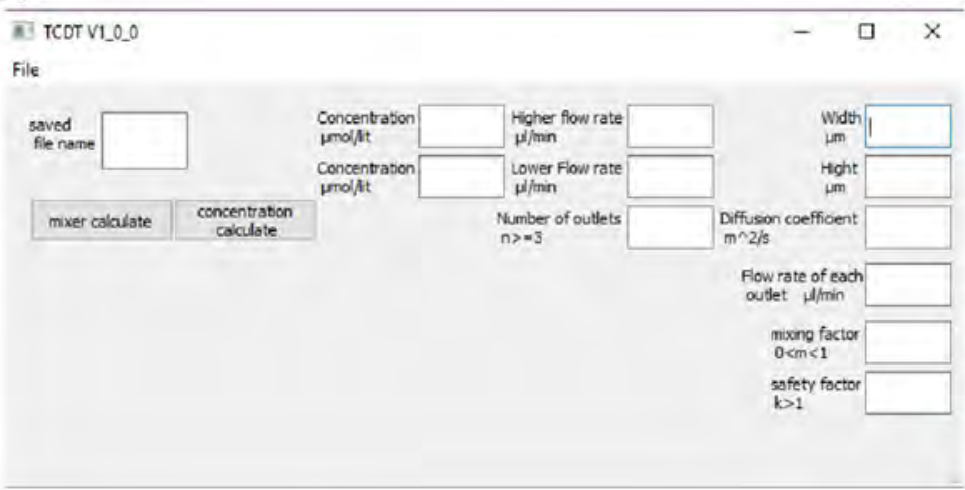

B

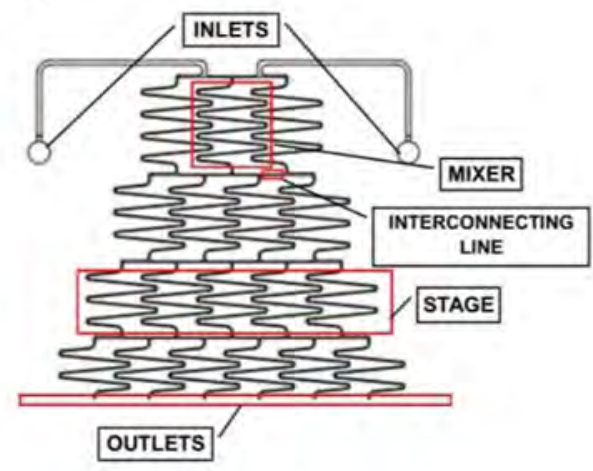

C

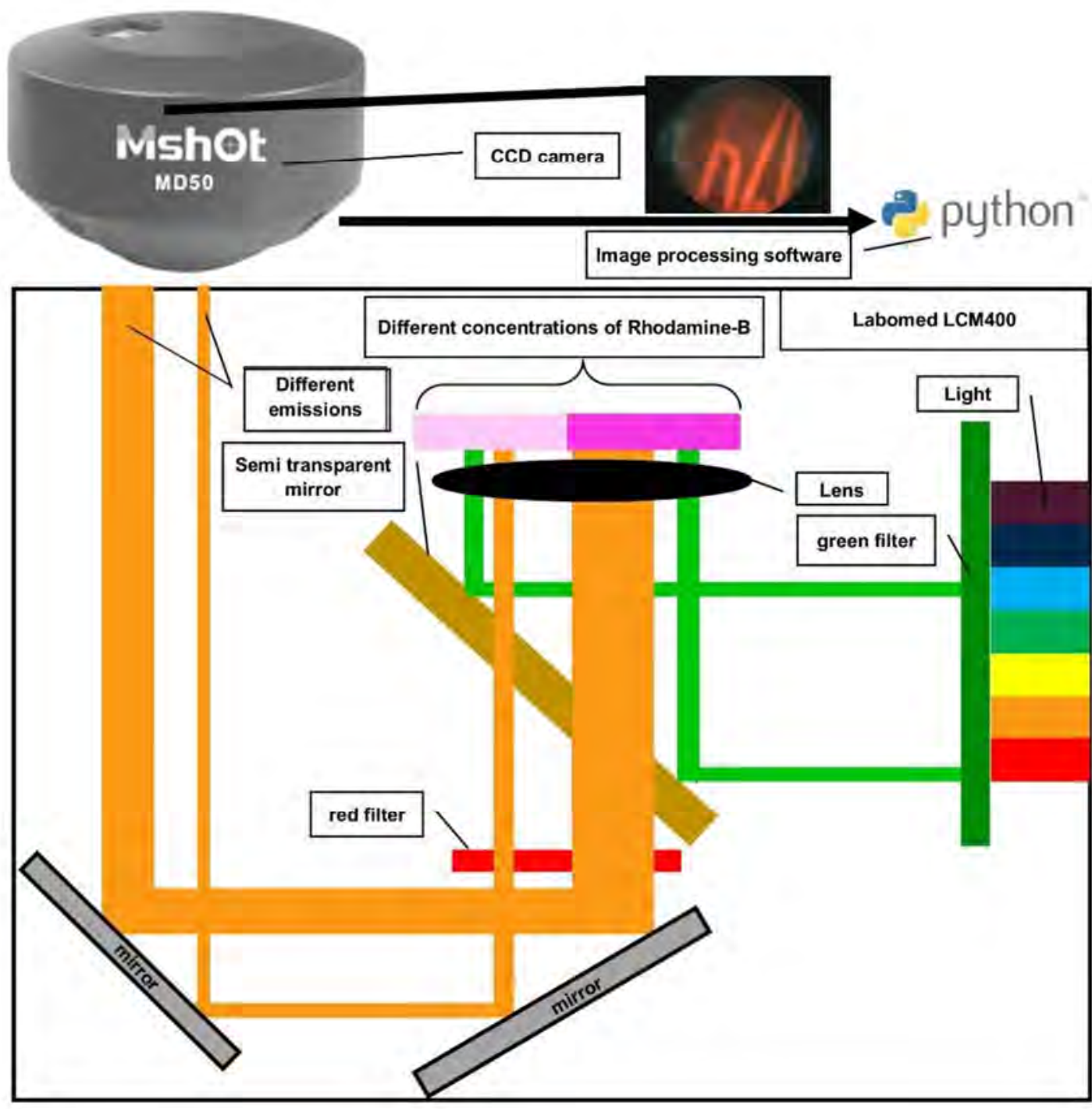

Fig. 1. A) The user interface made for the design tool. B) Schematic representation illustrating different parts of the device. C) Schematic of concentration measurement system. 


\section{Results and discussion}

The mixing performance of three CGGs was studied to validate the proposed model. To this aim, the actual output concentration of each inlet was compared with the predicted output concentration based on the proposed numerical simulation. For each case, the device mold was fabricated using a different fabrication method. The TCDT was used to design these three cases, and the output geometries were used for the subsequent fabrication.

\subsection{First case -- four-output concentration}

The first device under investigation is a CGG that receives single drug and diluter and gives four different drug doses at the outlets (Fig. 2A). The chip mold was fabricated using 3D-printing as mentioned previously. For this chip, the mixing performance was evaluated by measuring the fluorescence emission at each outlet followed by comparing the results with the outlet concentration predicted by the numerical simulation.

The chip was designed based on an input flow rate of $1.25 \mu \mathrm{l} / \mathrm{min}$ for each outlet, resulting in an overall flow rate of $5 \mu \mathrm{l} / \mathrm{min}$. The dimension of all the microchannels was $40 \mu \mathrm{m}$ in height and $150 \mu \mathrm{m}$ in width, set based on the best resolution of the 3D-printer, the datasheet-recommended design points and the analytical model of the device. The design and simulation were carried out based on the diffusion coefficient of $5 \times 10^{-10} \mathrm{~m}^{2} / \mathrm{s}$, which is lower than most of the conventional drugs and diluters commonly used in CGGs (Toh et al., 2014). Grid study was performed and achieved mesh independency (Fig. 2C) using $5.8 \times 10^{6}$ unstructured tetrahedral elements. The results of the numerical simulation of the device for different flow rates are shown in Fig. 2D. As these results show, the output concentration of the device deviates from the designed value using corrected form 
of Eq. 4 (labeled as "analytic model" in the figure) when the flow rate exceeds the design value of 5 $\mu 1 /$ min because the mixing does not occur completely. This incomplete mixing can be attributed to the insufficient length of the mixing channel.

The performance of the device was experimentally evaluated for different flow rates. As shown in Fig. 2E, the output concentration of the device obeys the simulation results for the flow rate of 5 $\mu 1 /$ min. The output concentration in the flow range of the design is linear; however, by increasing the flow rate, the concentration profile deviates from linearity. As mentioned before, this effect is due to the incomplete mixing that occurs in the presence of the high flow rate within the mixers. To investigate the reproducibility of the tests, all experiments were conducted three times, and the data are shown with error bars in Fig. 2E. 
A

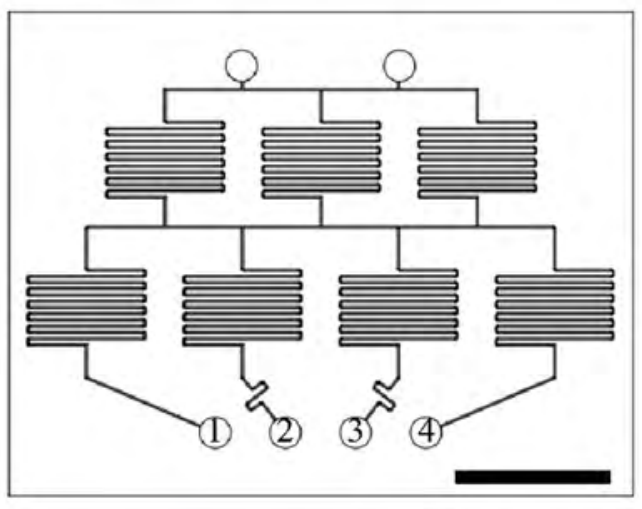

C

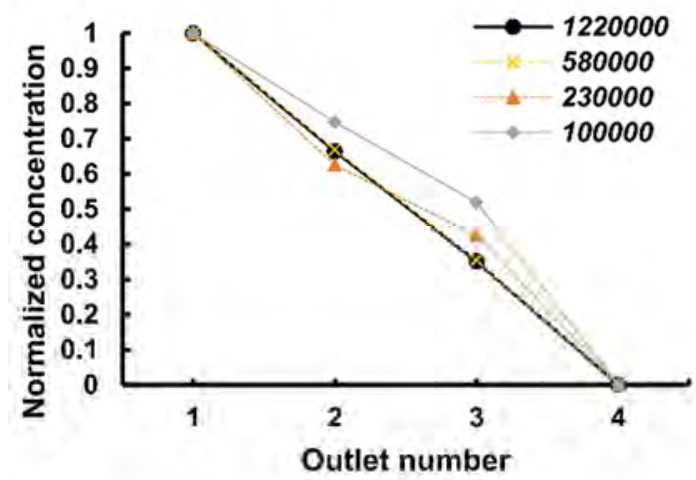

E

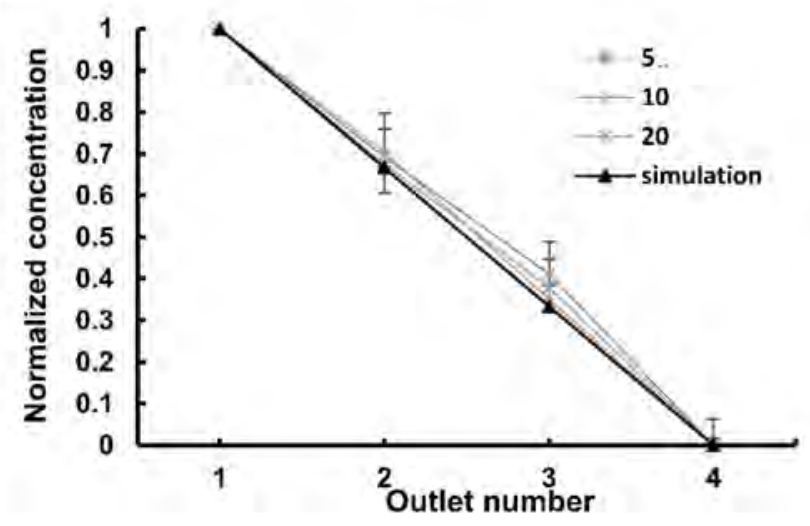

B
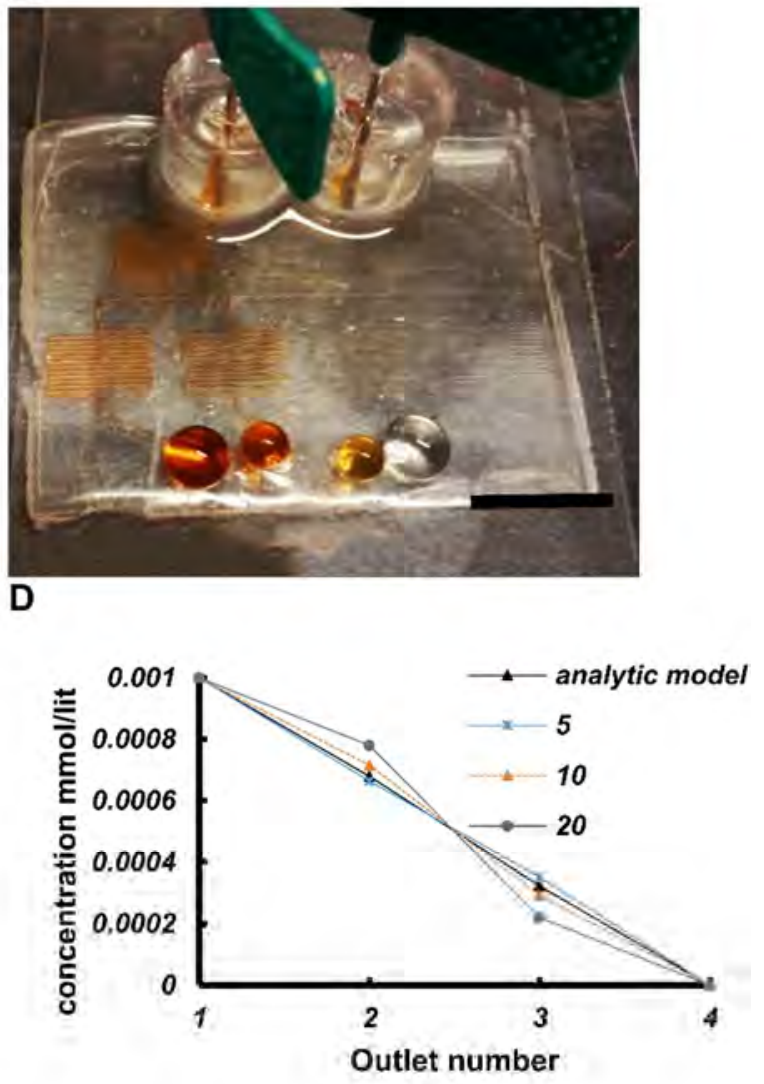

F

\begin{tabular}{|c|c|c|c|c|}
\hline Outlet number & 1 & 2 & 3 & 4 \\
\hline Flow rate & \multicolumn{4}{|c|}{ Uncertainty } \\
\hline \multirow{2}{*}{$5 \mu \mathrm{min}$} & 0.00 & 0.03 & 0.03 & 0.07 \\
& 0.09 & 0.09 & 0.09 & 0.02 \\
\hline \multirow{2}{*}{$10 \mu \mathrm{l} / \mathrm{min}$} & 0.03 & 0.09 & 0.06 & 0.06 \\
& 0.09 & 0.04 & 0.08 & 0.03 \\
\hline \multirow{2}{*}{$20 \mu \mathrm{l} / \mathrm{min}$} & 0.05 & 0.07 & 0.08 & 0.06 \\
& 0.01 & 0.09 & 0.03 & 0.08 \\
\hline
\end{tabular}

Fig. 2. (A) The shape of the designed mold (Scale bar: $10 \mathrm{~mm}$ ). (B) The fabricated device. Red food dye was used in the channel, and a scalp needle was used to inject the flow to the device (Scale bar: $10 \mathrm{~mm}$ ). (C) Grid study of the simulation for a flow rate of $5 \mu \mathrm{l} / \mathrm{min}$. The numbers under the horizontal axis are the outlet numbers shown in Fig. 2A. (D) Simulation of the device performance for different flow rates. (The unit of the numbers in the legend is in $\mu 1 / \mathrm{min}$ ). The term "analytic model" refers to the corrected form of Eq. 4 based on the concentration outputs that the device was designed to generate. (E) Experimental colorimetric results of the output concentration. The error bars represent the test result range. The outlet numbers are mentioned in Fig. 2A. (The unit of the numbers in the legend is $\mu \mathrm{l} / \mathrm{min}$ ) (F) The values of the uncertainty bars in Fig. 2E. 


\subsection{Second case -- eight-output concentration device}

The second CGG studied in this research is the eight-concentration single-drug device. This device mold was fabricated using CNC micromilling. The device was designed using the TCDT and was simulated using a CFD code. The resulted concentration of the device was evaluated using fluorescent emission intensity measurement.

\subsubsection{Simulation results}

Fig. 3A shows the device design. The large dimensions of the device $(55 \mathrm{~mm} \times 50 \mathrm{~mm})$ are due to its high flow rate and the fabrication method. The device was designed based on the flow rate of $5 \mu 1 / \mathrm{min}$ for each output and eight concentrations that resulted in a total amount of $40 \mu \mathrm{l} / \mathrm{min}$. To improve the fluorescence imaging of the device, eight reservoirs were placed after each outlet of the device. The oblique placement of the channels is for reducing the width of the device because in this configuration, the mixers can be placed closer to each other (Fig. 3A).

As shown in Fig. 3A, the mixer length decreases as the liquid flows downstream in each stage of the device. This occurs because the number of mixers increases and the flow rate decreases in the mixers in the last stages. This effect was considered in the TCDT to keep the device dimensions as small as possible. In this device, the first stage mixer is 3.5 times longer than the last stage.

Fig. 3B depicts the CFD simulation results of the output concentrations for different flow rates. Increasing the flow rate to by one order of magnitude led to a reduction in the mixing performance. Increasing the flow rate in the channel does not allow enough time for diffusion before exiting the mixing channel. This effect is of utmost importance in the design of the mixers. Therefore, the length and the dimensions of mixers should be designed according to the flow rates within the channels to allow complete mixing, Eq. (4). 
Most of the CGGs produce linear concentration gradients of drug. However, producing linear concentration gradients does not necessarily lead to the desired results. The non-linear concentrations are sometimes more beneficial because, in dilute concentrations of drug, small changes in the amount of the drug can result in a considerable change in concentration. Conversely, in high concentrations of drugs, small changes may not have sensible changes (Toh et al., 2014). Therefore, nonlinear output concentration can result in more comprehensive concentration changes in drug screening.

TCGGs can produce nonlinear concentration gradients by changing the ratio of the flow rate of the input fluids (Toh et al., 2014). The predicted nonlinear concentration profile in TCGGs can be obtained more precisely if the number of the outputs is high enough (Keenan and Folch, 2008). Fig. $3 \mathrm{C}$ illustrates the response of the device predicted by the TCDT for different ratios of the flow rate in the input. By increasing the flow rate ratio to 3, the output concentration of the outlets nearest to the high flow rate input (e.g., outlet number 8 and 7 for 1:3 drug to diluter ratio) approaches the concentration of the higher flow rate. There is no outlet delivering the fluid with lower flow rate purely. Therefore, its highest normalized concentration is 0.75 (Fig. 3C). The nonlinear concentration output has a sharper gradient than the linear one. For example, for the diluter to drug flow rate ratio of 1.9, the normalized drug concentrations are shown in Fig. 4A. By comparing the concentrations of the outlets of non-linear experiment to linear experiment (all except 1:1 to 1:1 in Fig. 3C or nonlinear ones to linear), it can be seen that by this combination of the drug and the diluter, a broader resolution of drug is provided. Especially for low concentrations at which small changes lead to large differences. To present the difference better, each output concentration was divided to the next one (concentration of outlet number 3 divided to number 2, number 4 divided to number 3 , and so on). As shown in Fig. 4A for 1:1 and 1:1.9 of Rhodamine-B to diluter, the concentration ratios of the nonlinear combination provides a wider range of concentration ratios because it changes almost an 
order of magnitude (6 times) in the third outlet relative to the second. Therefore, this combination could be used for drug research more effectively as its range of concentration changes is more comprehensive. 
A

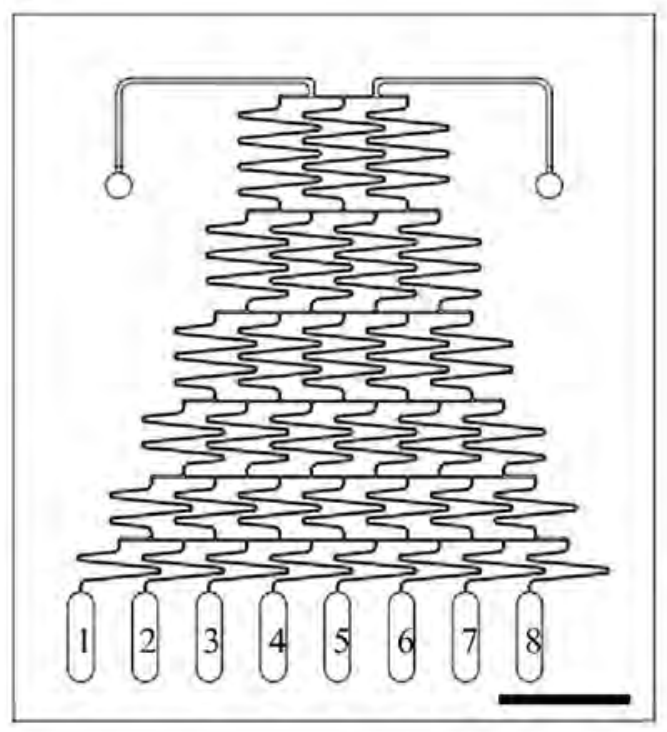

B

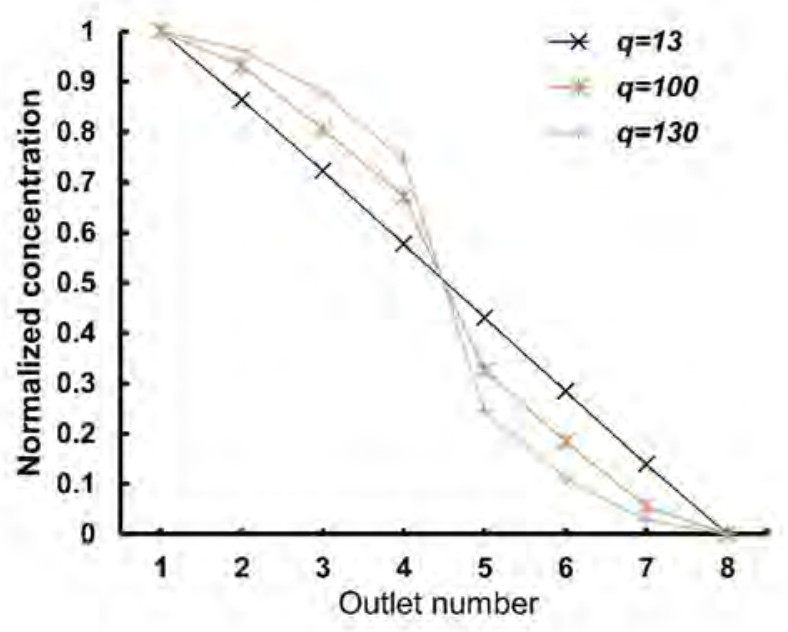

C

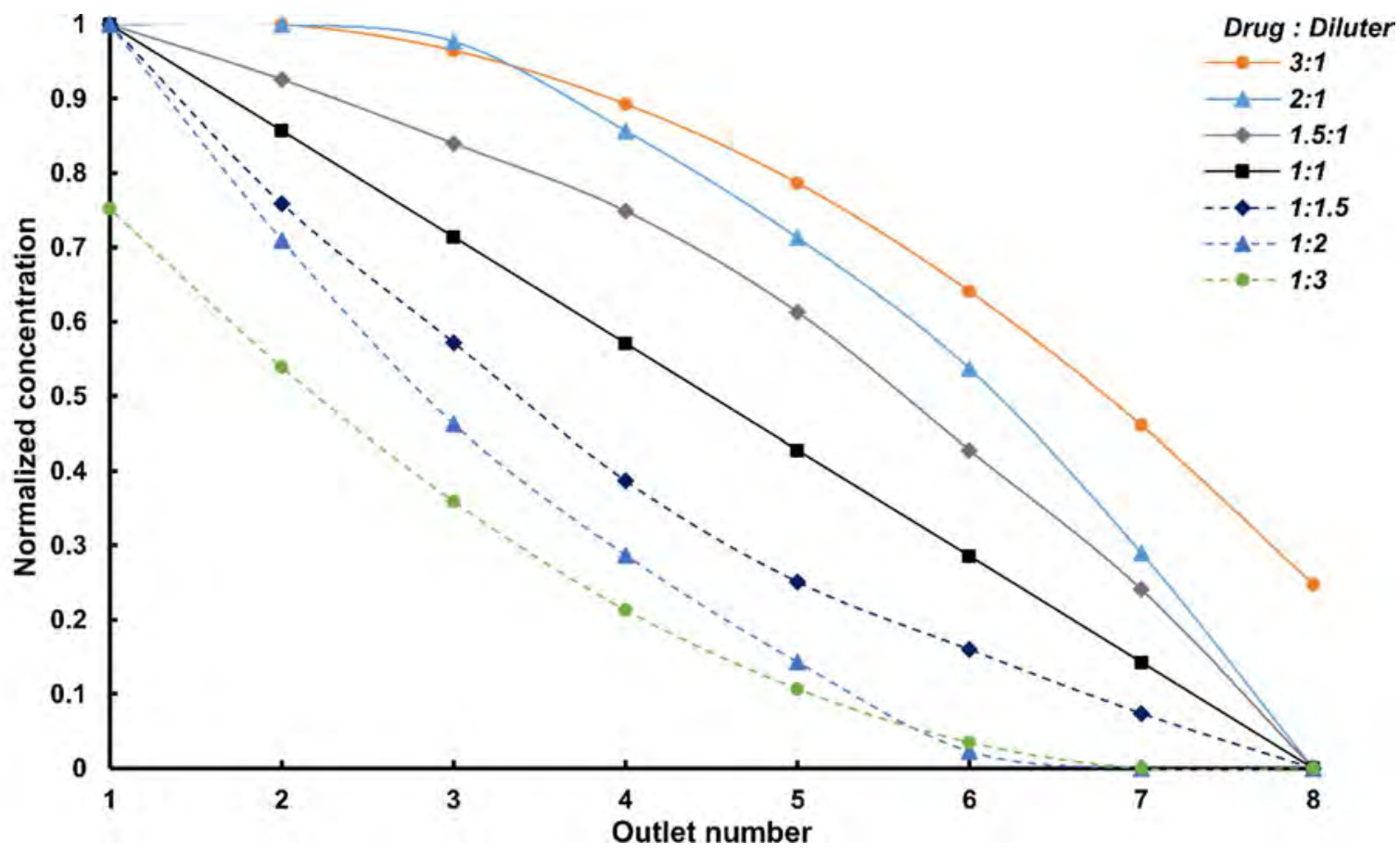

Fig. 3. (A) The schematic of the mold (Scale bar: 10mm). (B) CFD results of the device performance in terms of normalized drug concentration for different flow rates at the outlets (outlet numbers are shown in (A)). (The unit of the numbers in the legend is $\mu 1 / \mathrm{min}$ ). (C) Normalized concentration for different flow rate ratios of drug to diluter at the inlets, obtained by CFD simulation. 
A

B

\begin{tabular}{|c|c|c|c|c|c|c|c|c|}
\hline \multirow[t]{4}{*}{ 1:1.9 } & \multicolumn{8}{|c|}{ Normalized Concentration } \\
\hline & 0 & 0.01 & 0.06 & 0.16 & 0.31 & 0.49 & 0.72 & 1 \\
\hline & \multicolumn{8}{|c|}{ Ratio } \\
\hline & $\operatorname{lnf}$ & 6 & 2.6 & 1.9 & 1.6 & 1.5 & 1.4 & \\
\hline \multirow[t]{4}{*}{ 1:1 } & \multicolumn{8}{|c|}{ Normalized Concentration } \\
\hline & 0 & 0.14 & 0.29 & 0.42 & 0.57 & 0.70 & 0.86 & 1 \\
\hline & \multicolumn{8}{|c|}{ Ratio } \\
\hline & Inf & 2.1 & 1.5 & 1.3 & 1.2 & 1.2 & 1.2 & \\
\hline
\end{tabular}

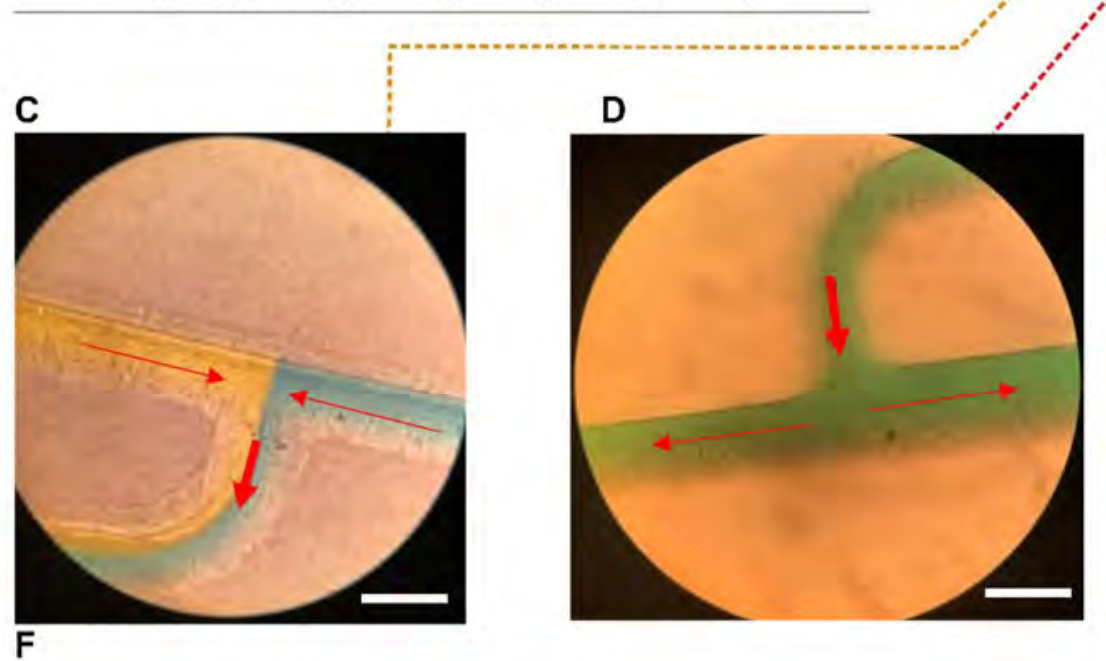
E
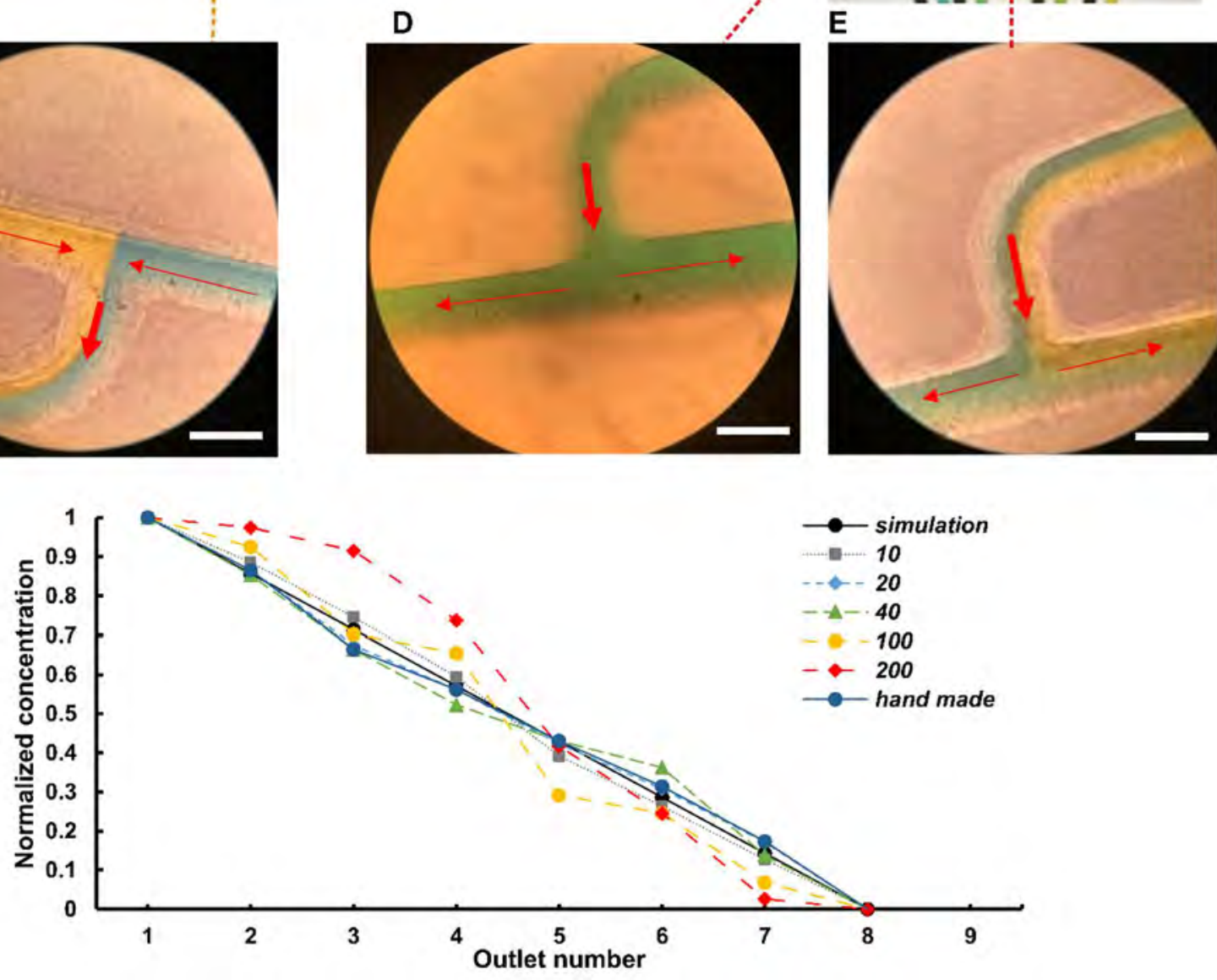

Fig. 4. (A) The output concentration and its ratio for flow ratio of Rhodamine-B to diluter 1:1.9 and 1:1 (B) Connection of the CGG for 4 output concentrations (Scale bar: 10mm). (C) The inlet junction of the first mixer of the device; the interface between the streams is noticeable. The arrows indicate the flow direction (Scale bar: $0.15 \mathrm{~mm}$ ) (D) The outlet of the first mixer of the device; the border vanishes due to complete mixing. (Arrows show the flow direction) (Scale bar: $0.15 \mathrm{~mm}$ ). (E) The outlet of the first mixer of the device; the interface has not vanished due to incomplete mixing. The arrows indicate the flow direction (Scale bar: 0.15 $\mathrm{mm}$ ) (F) The output concentration of the device for different flow rates in the experiment, the simulation, and the fabricated CGG. As the flow rate increases, the output concentration is less accurate (The error bars are omitted to allow visibility of all points) (The unit of the numbers in the legend is $\mu 1 / \mathrm{min}$ ). 


\subsubsection{Experimental performance analysis}

One of the capabilities of TCGGs is producing a variety of concentrations. In these devices, the performance is dependent on the pressure drop and the resistance of the channels. That means, any change in the resistance results into a change in the output flow rate and concentration distribution amongst the outlets of the device. Thus, it is highly critical to design and manufacture the channels and the downstream chip that delivers the output fluid of the device such that the pressure drop remains equal among parallel channels. The undesired outlet will be closed to use a smaller number of outlets. For example, if one of the outlets is closed, the device will act exactly similar to a 7-outlet device. This effect was investigated in connection with a cell culture chip depicted in Fig. 4B. As can be seen, the device has created the desired color concentrations in both cases.

As mentioned above, the most crucial part of designing a CGG is the mixer. The inlet and outlet of a mixer are shown in Figs. 4C and D, respectively. At the inlet of the mixer, the two parallel flows are not mixed and have a visible interface. However, the interface vanishes, and mixing occurs completely downstream. As can be seen in Figs. 4C and D, the roughness on the channel wall created during fabrication of the mold, although not initially considered as a design parameter, improves the function of the device as it enhances mixing. If the mixer does not work perfectly, the interface between the streams does not vanish at the exit (Fig. 4E).

Two experiments were performed to measure the effect of changing the flow rate on the generation of linear and nonlinear concentrations. The fabricated device was connected to a syringe pump, and the output concentration was evaluated using fluorescence intensity measurement for different flow rates. By inducing the same flow rate of Rhodamine-B and water, the output concentration was linear for the inlet flow rate of $20 \mu \mathrm{l} / \mathrm{min}$ at each inlet of the CGG. For a higher 
discharge, the resulting concentration will slightly deviate from the desired value. For this test, the flow rate was set at 10,20,40,100,200 $\mu \mathrm{l} / \mathrm{min}$ at each inlet, and the results of the light intensity of the fluorescence material are shown in Fig. 4F.

As shown in Fig. 4F, the device operates with an error as low as 5\% up to the designed discharge of $20 \mu \mathrm{l} / \mathrm{min}$ at each inlet, but at a higher discharge, the result slightly deviates from the ideal (i.e. linear) concentration gradient. This effect was similarly predicted by the simulation shown in Fig. 4F. However, the deviation in the simulation was slightly lower than the experimental one, which was possibly due to the limitations in the fabrication accuracy as well as small bubbles trapped in the device. Bubbles will change the pressure drop in the channels that affect the flow rates. For instance, at very small flow rates, there is a slight deviation from the desired value due to the small pressure drop, and because there are some bubbles in the channels that result in unequal pressure drop between the parallel channels and altered output concentration. 
A

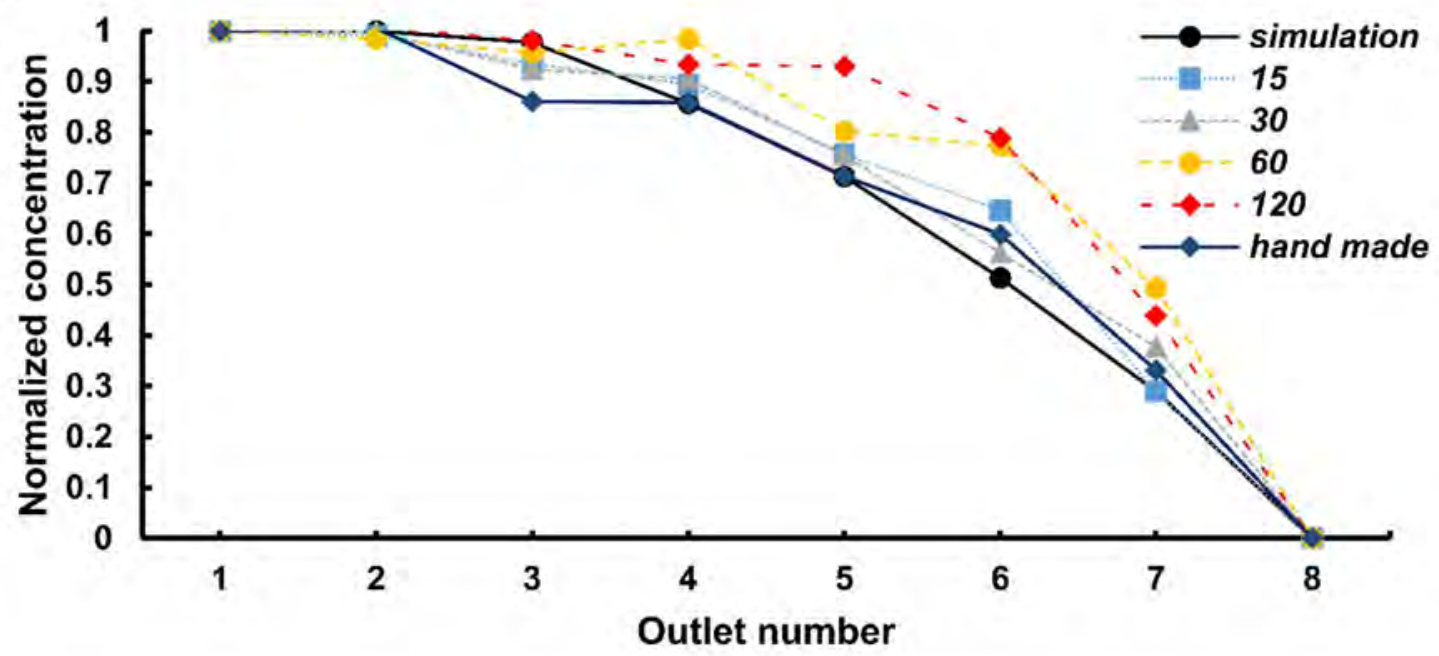

B
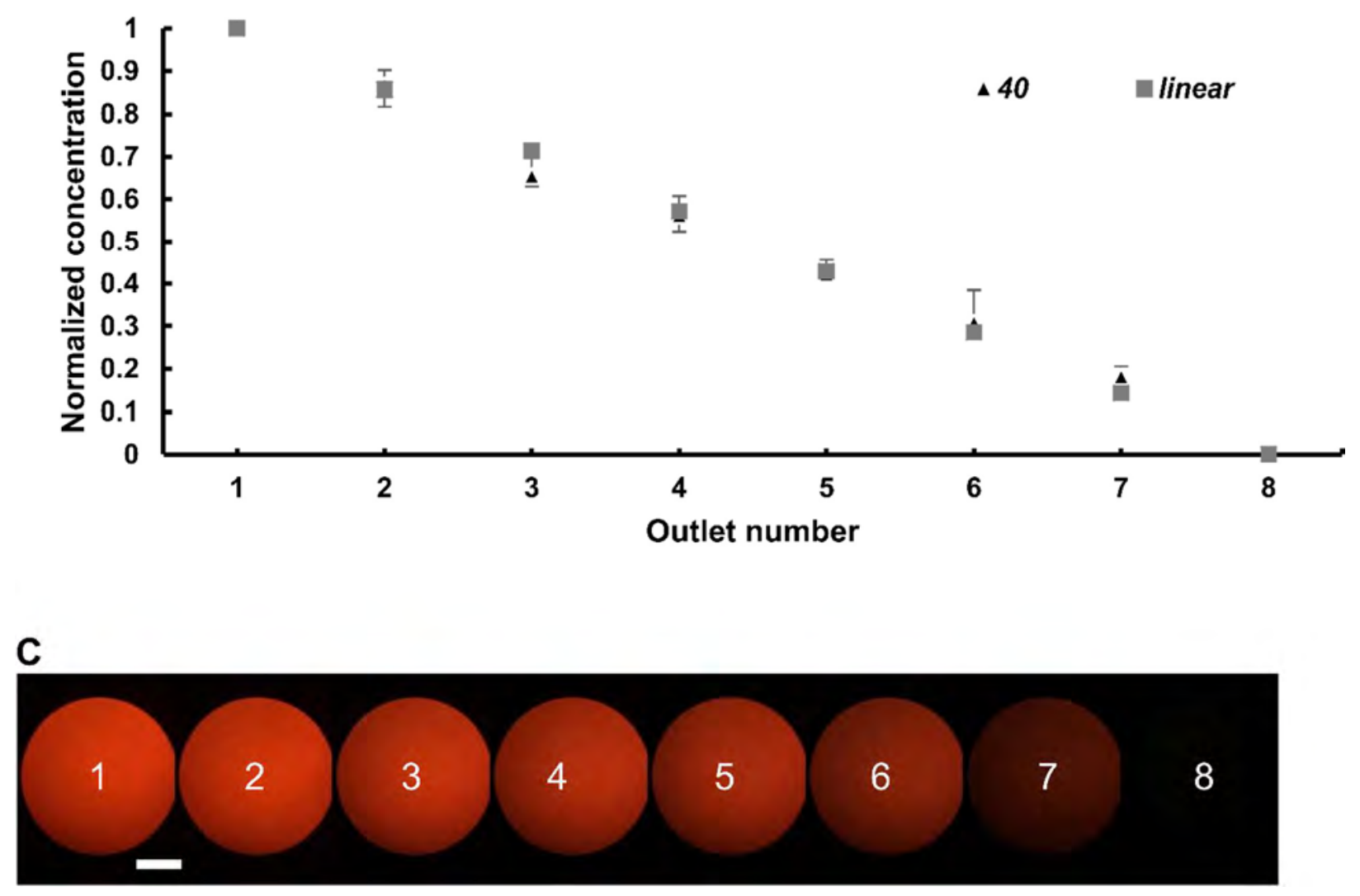

Fig. 5. (A) The output concentration of the device with the Rhodamine-B: water flow rate ratio of 2:1 for different flow rates. (Numbers in the legend are in $\mu 1 / \mathrm{min}$ ) (B) The output concentration of the device for a flow rate of $40 \mu \mathrm{l} / \mathrm{min}$. (The range of the results of the three experiments is shown as the uncertainty bars) (The unit of the numbers in the legend is $\mu 1 / \mathrm{min}$ ). (C) The images captured from the CGG outlets with fluorescence microscopy (Scale bar: $0.05 \mathrm{~mm}$ ). 
The second experiment was designed to examine the effect of the difference in flow rates at the inlets on the device inputs. In this test, the flow rate of one of the inputs is two times larger than the flow rate at the other input. As obtained from the simulations shown in Fig. 5A, by changing the relative flow rate of the inputs of the device, the distribution of the concentration gradient at the outlet varies from linear to nonlinear with a sharper gradient. This effect is caused by the asymmetry created at the entrance, and consequently, in all channels. To perform this test, Rhodamine-B was used with the same concentration as in the first test. Rhodamine-B flow rate was twice as much as water's, and imaging took place under steady-state conditions. Normalized concentration graphs are shown in Fig. $5 \mathrm{~A}$.

With increasing ratios of Rhodamine-B to water flow rate at the inputs, the concentrations of the outlets near the rhodamine-B side increase and approach each other (Fig. 5A). This feature is especially useful for drug studies that do not specify a concentration level, as it can be used to test a greater range of concentrations and observe the effects. Increasing the input flow rate disrupts the diffusion effect of the channels. This effect causes a great difference in the output concentration, similar to the linear concentration. Fig. 5B shows the results of the test performed for a flow rate of $40 \mu \mathrm{l} / \mathrm{min}$. The images of the outlets are shown in Fig. 5C. The error bars are smaller at lower Rhodamine-B concentrations, which shows that the optical noises are less for small Rhodamine-B concentrations. In addition, as the data are normalized relative to the first and last output concentrations, the error bars are omitted in outlets 1 and 8 . 


\subsection{Third case - Multi-drug chip}

The multi-drug chip was designed using the TCDT for a flow rate of $15 \mu 1 /$ min from each inlet and diffusion coefficient of $5 \times 10^{-10} \mathrm{~m}^{2} / \mathrm{s}$. The channel cross-section dimensions are $50 \times 50 \mu \mathrm{m}$. The device was simulated using the CFD code and manufactured using a photolithographic mold and soft lithography. The output concentration of the device was measured using fluorescence emission measurement and color measurement.

The device has three inlets in the center and twelve outlets around the chip (Fig. 6A). This orientation of the mixers facilitates the investigation of the effect of three drugs. Fig. 6B illustrates the qualitative concentration distribution using food dyes at the outlets. Figs. $6 \mathrm{C}$ and $\mathrm{D}$ show the inlet of the same mixer for color (C) and Rhodamine-B (D); the interface between the two streams is visible in both pictures. 
A

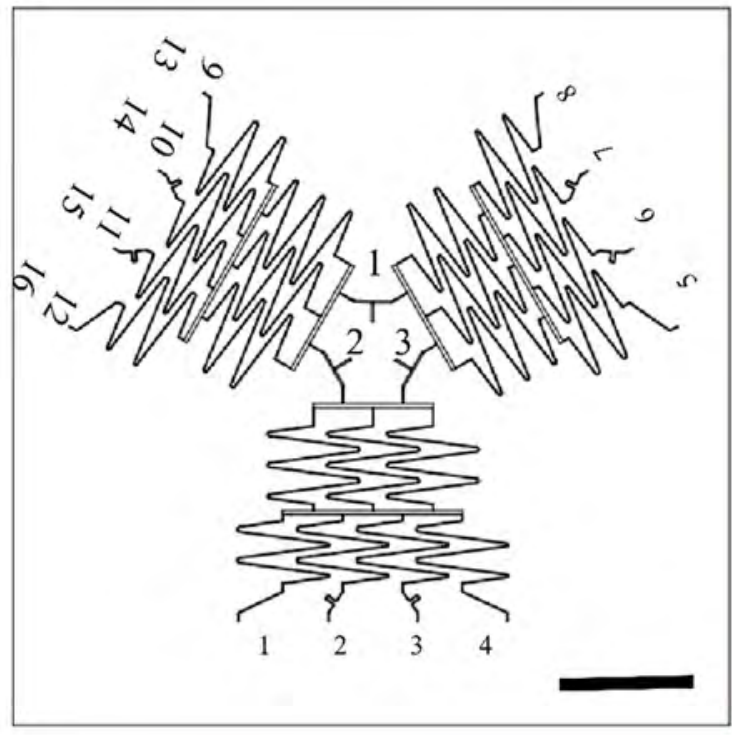

C

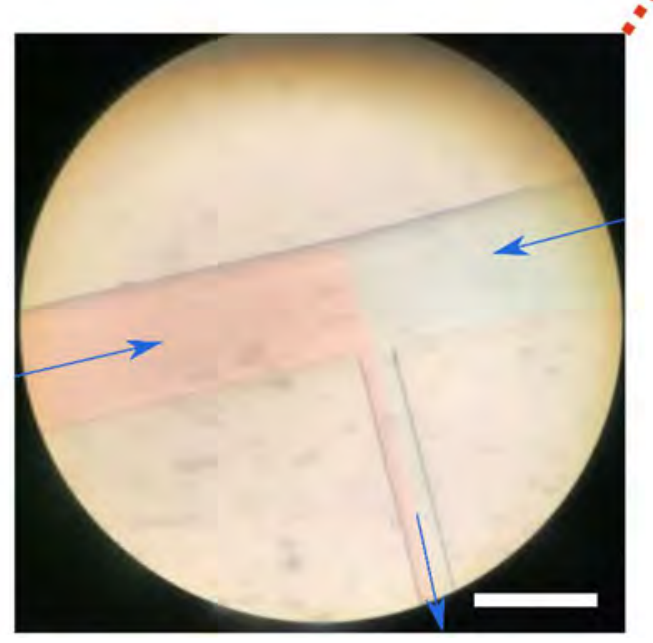

B
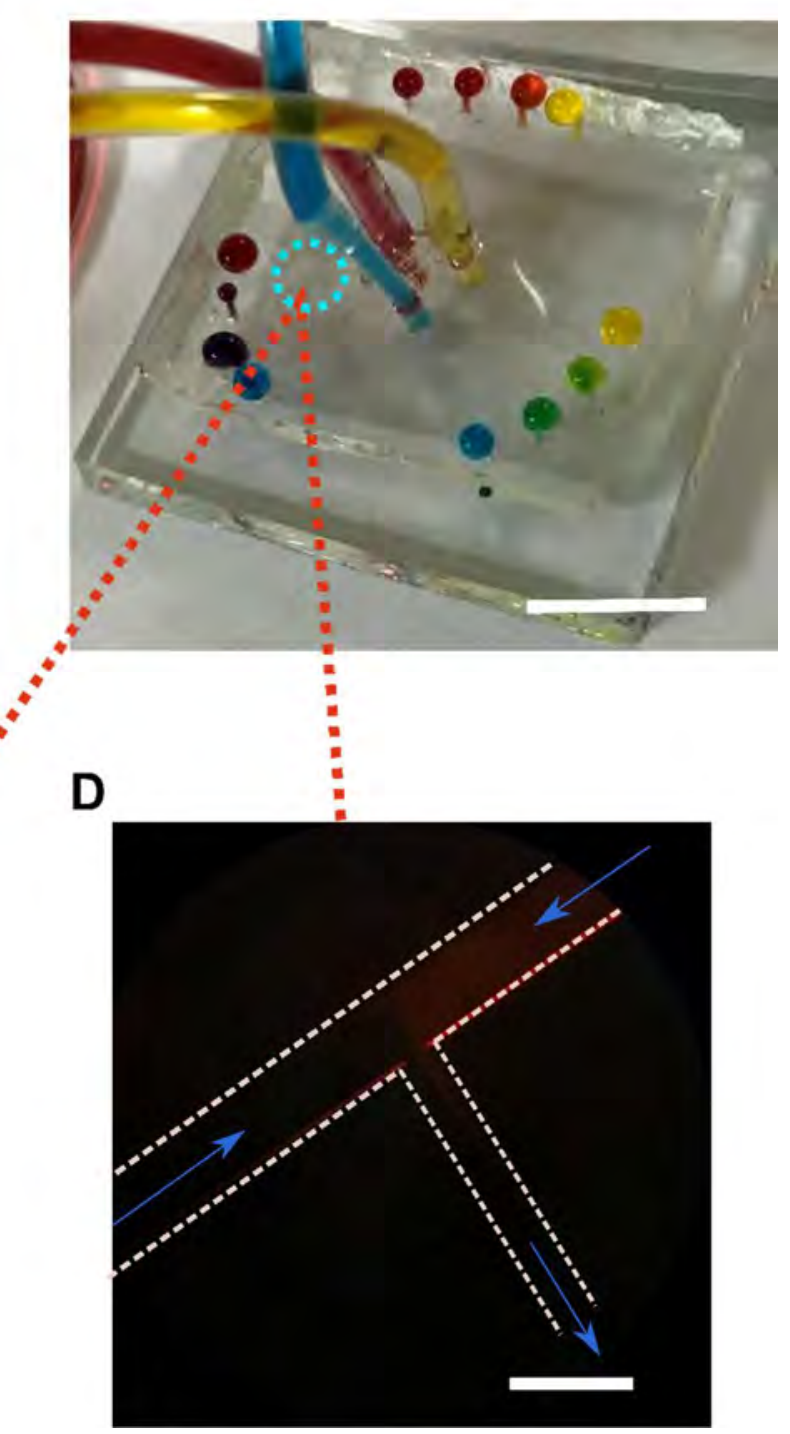

Fig. 6. (A) Overal schematic of the device's mold (scale bar: $10 \mathrm{~mm}$ ). (B) The color gradient at the device outlets (Scale bar: 10mm) (C \& D) The inlet of the first mixer of the device visualized using food dye and fluorescent emission. (The white dashed lines represent the edge of the channels) (Arrows show the direction of flow) (Scale bars: $0.15 \mathrm{~mm}$ )

\subsubsection{Multi-drug multi-concentration method}

In Figs. 7A, 7B and 7C, the output concentration of the device for a single drug, two drugs, and three drugs were investigated. Using this device with two drugs, the effects of each drug individually, as well as the two drugs simultaneously could be checked. By adding a third drug, it is not possible to 
check the function of the drugs individually. In contrast, the two-by-two interactions of the three drugs are examined.

Figs. 7A, B and C show the simulated output concentrations of the device for the design flow rate. Fig. 7A is the output concentration of the device for a single drug from one inlet and diluter from two inlets. This combination does not have any new features compared to the single-drug chip. Fig. 7B shows the results of adding two drugs from two inlets (no matter which one) and the diluter from the third one. This combination can produce the concentration gradient of two drugs with diluter (outlets 5-8 and 9-12) and the combination of the two drugs (outlets 1-4). Using this method, it is possible to compare the effect of two drugs with a single drug. Fig. 7C demonstrates the two-by-two combination of three drugs. With this method, the effect of the drug combinations (outputs 2, 3, 6, 7, 10, and 11) and also single drug effect (outputs $1,4,5,8,9,12$ ) can be examined. This device allows for finding the best combination of the drugs first. Subsequently, the single-drug chip finds the best concentration of the drugs. In addition, this device can be used to investigate the effect of two-bytwo combinations of three drugs relative to each other and find the best drug concentration by using the single-drug chip.

The method mentioned above is named as Multi-Drug Multi-Concentration chip (MDMC) for personalized medicine. MDMC on a patient could be an off-the-shelf (Mark et al., 2012) personalized medicine approach for patients with more than a single drug option. The method is based on the fact that for each person and drug combination, there is a need for designing a specific CGG based on the requirements mentioned before. Thus, using the TCDT and the MDMC method together can produce the desired results applicable in personalized medicine, especially because it is compatible with the low-cost rapid prototyping methods such as 3D printing. 


\subsubsection{Experimental performance analysis}

Because there are three different inputs in the multi-drug sample, Rhodamine- $\mathrm{B}$ and distilled water are not adequate for the performance analysis. The experiment was divided into two stages. In the first stage, the fluorescence material in one of the inlets such as inlet 2 and the diluter in the other two inlets entered the device (Fig. 8A), and concentration gradient formed in two sections of the three sections (outlets 1-4, 13-16 in Fig. 8A). In the second stage, by changing the device inputs (Rhodamin-B in inlet 1 and water in $2 \& 3$ ), the concentration gradient was formed in the third section (outlets 5-8) as well as outlets 9-12 (Fig. 8B), which were examined in stage 1 as 13-16. The generated concentrations were visualized in Fig. 8C.

Comparing the two stages of examination and normalizing the data could measure the concentration gradient of the device, Fig. 8C. In Fig. 8C, outlets 13-16 represent outlets 12-9 respectively in the second stage of the experiment and the concentrations of the outlets 5-8 are equal to those of the second stage. This experiment was designed to validate the mixing performance at a flow rate of $15 \mu \mathrm{l} / \mathrm{min}$. As illustrated, all the mixers have the same performance and react the same in the measurement of Rhodamine-B emission.

The above method is suitable for measuring the single concentration gradient, which was used to examine the mixing performance. The concentration was measured based on food dye intensity to determine the threshold flow rate below which the device output concentration remains unaffected. Food dye measurement makes it possible to investigate the CGG performance when more than two inputs with various drugs exist. For this purpose, the HSV method for color processing was used. Three colors, red, yellow, and blue, were introduced to the device. Because at each outlet, the color was a combination of two colors, e.g., red and blue, the relative color of each outlet was measured.

Fig. $8 \mathrm{D}$ depicts the concentration in each outlet are as follows: outlets 1 to 4 , concentrations of red 
compared with blue, outlets 5 to 8 concentrations of blue in comparison with yellow, and outlets 9 to 12 , concentrations of red in comparison with yellow.

Finally, the performance of the device at different flow rates was evaluated. Simulation results are compared with experimental data in Fig. 8D. The experimental output and the simulated output agree well for the design flow rate of $15 \mu 1 / \mathrm{min}$ at each inlet. However, increasing the flow rate leads to discrepancy between simulation and experiment. As mentioned above, this effect is due to incomplete diffusion resulting from the high flow rate. A critical feature in this device is that it was more sensitive to flow rate change than others represented before. A small increase in the flow rate above the designed values (from 15 to $25 \mu 1 / \mathrm{min}$ ), the mixing performance decreases rapidly (by more than 20\%). This effect is caused by the smoothing of the sharp edges of the channel when the device mold was made using photolithography techniques. In the micromachining and stereolithography techniques, the channel edges were not sharp and had some roughness. The roughness enhances mixing and improves the performance of the device. With photolithography, well-defined edges and smooth channel walls hinder convective mixing and increase the required mixing length. 
A

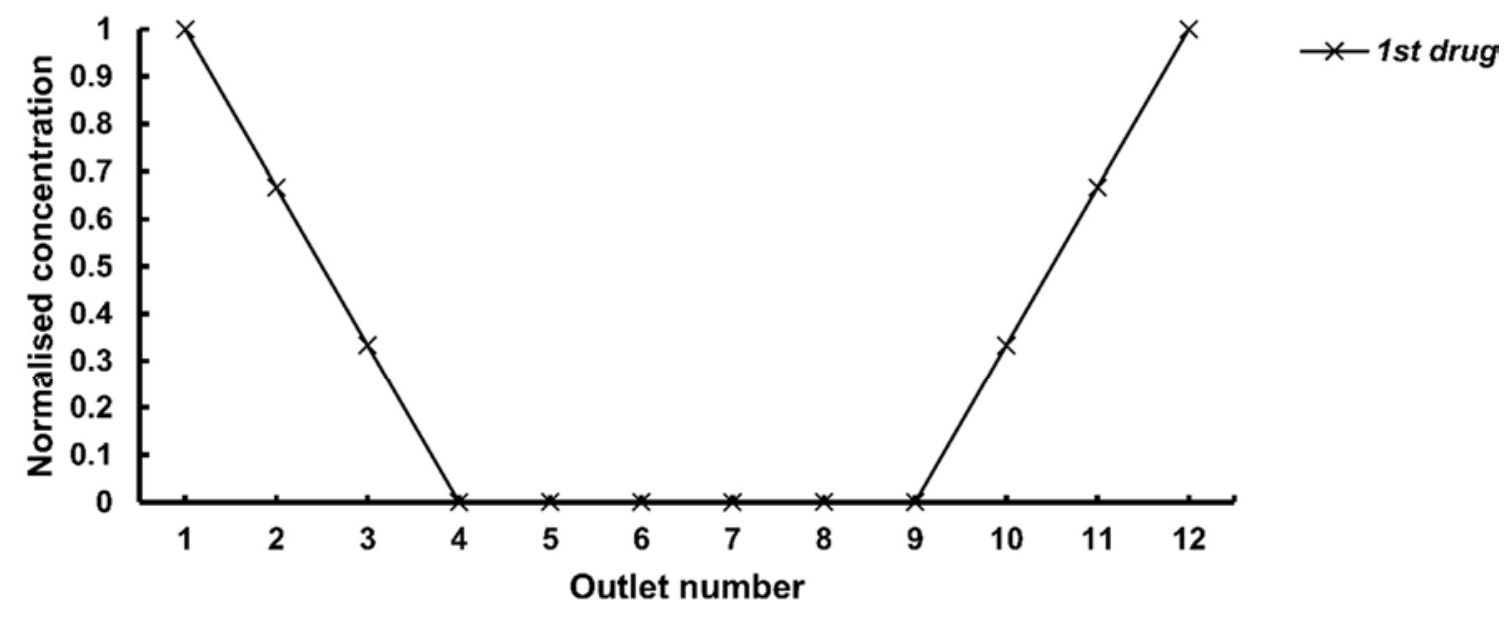

B

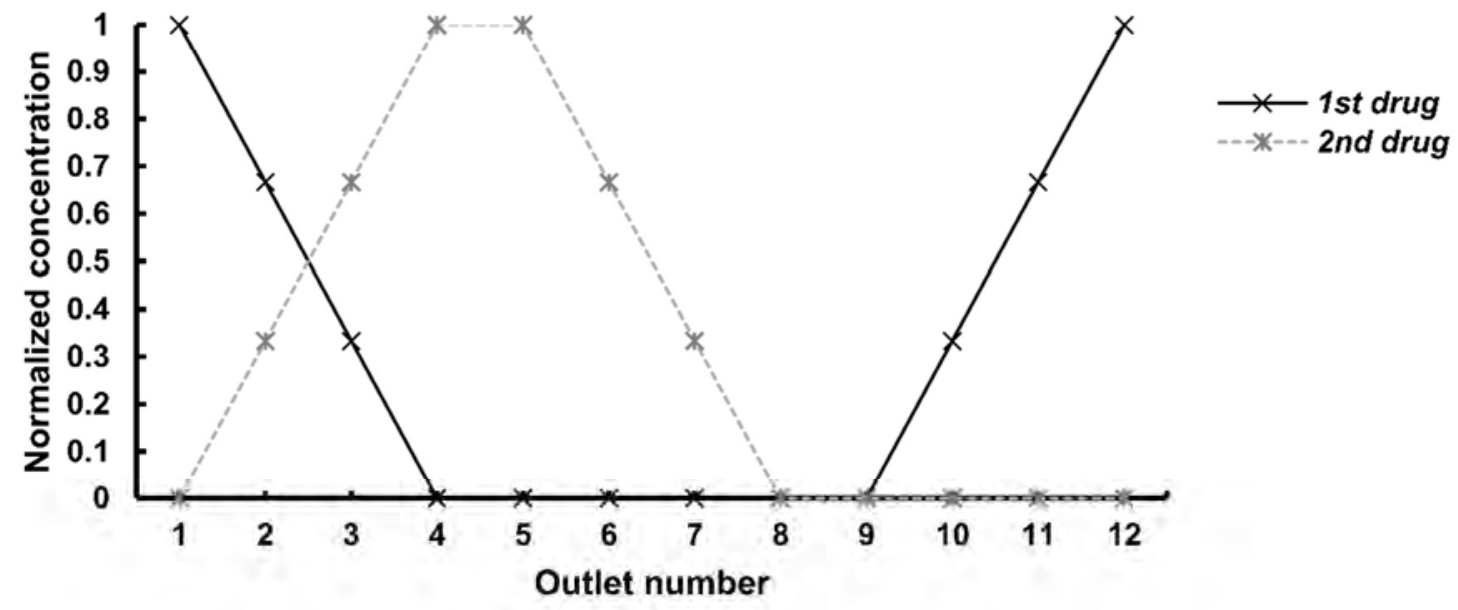

C

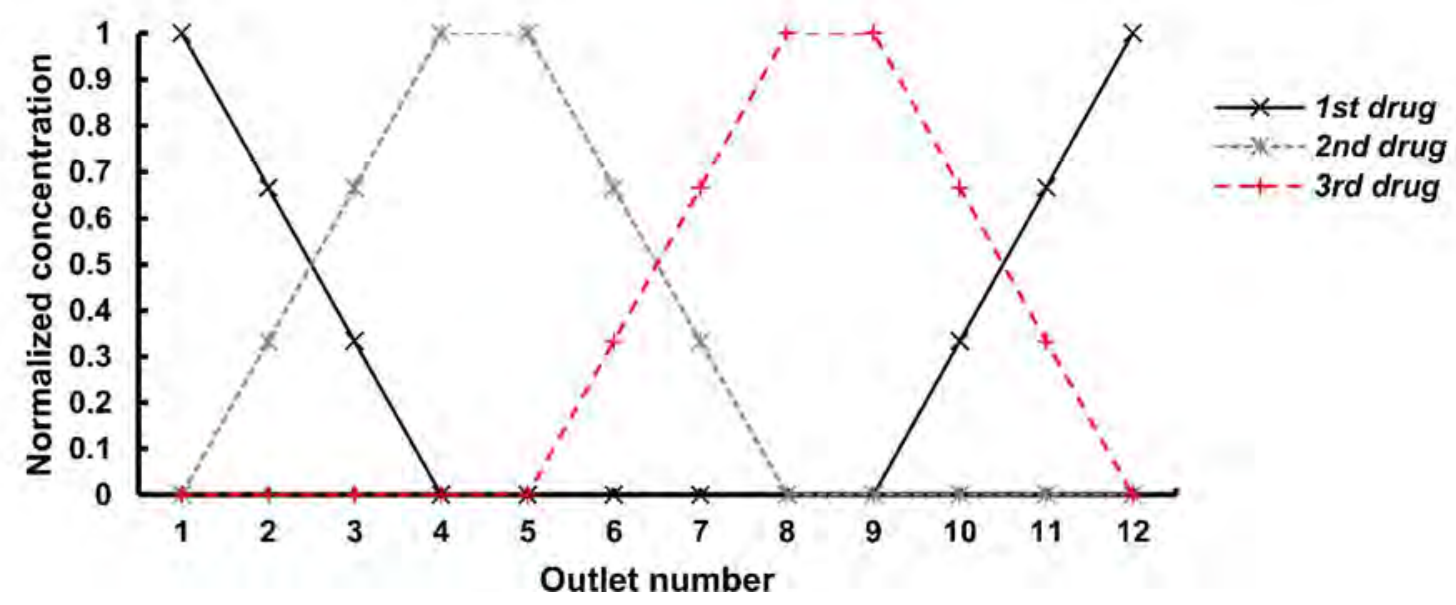

Fig. 7. Simulation results of the device (A) One-drug. (B)Two-drugs. (C) Three-drugs 
A

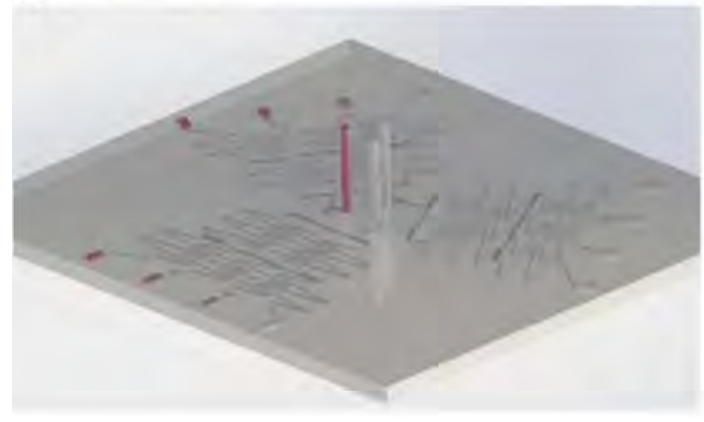

B

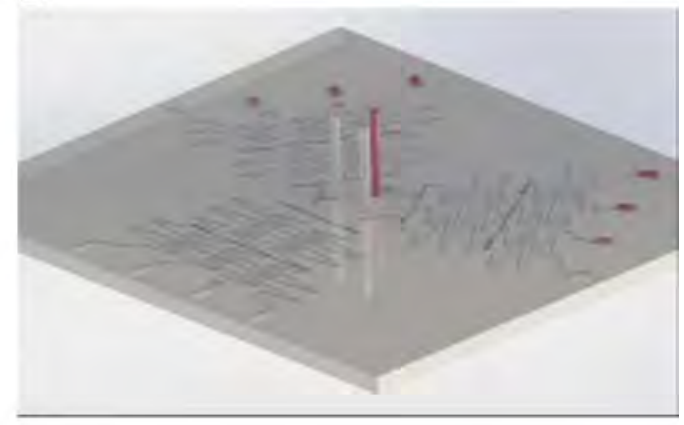

C

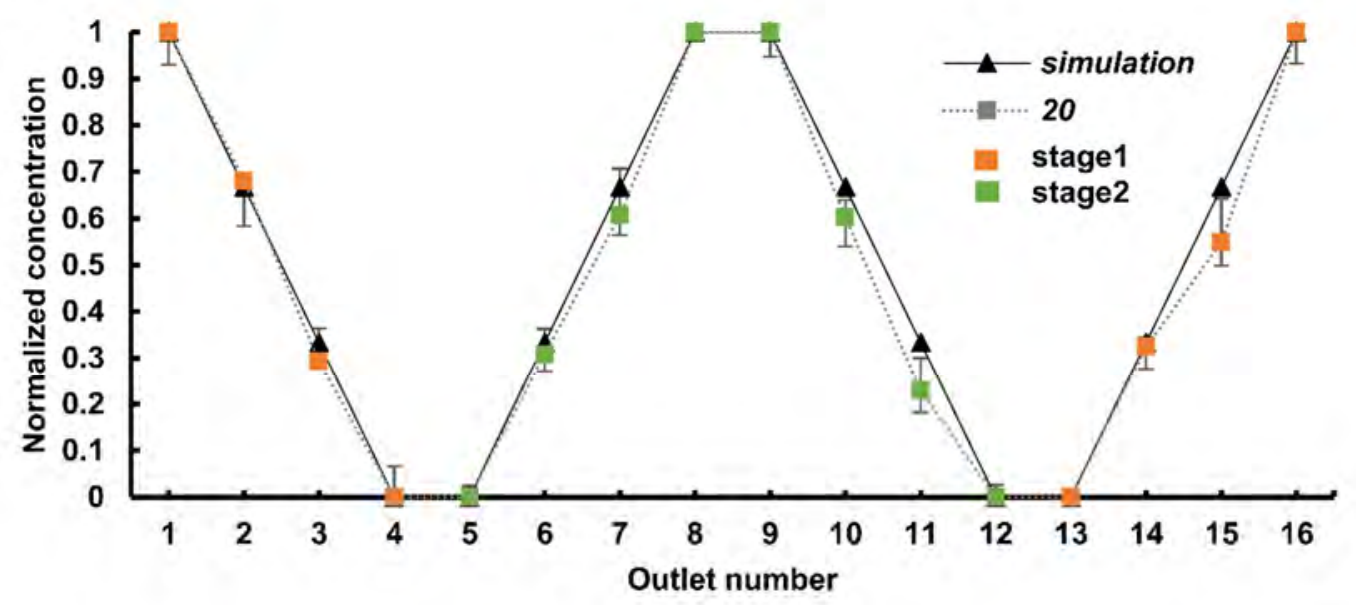

D

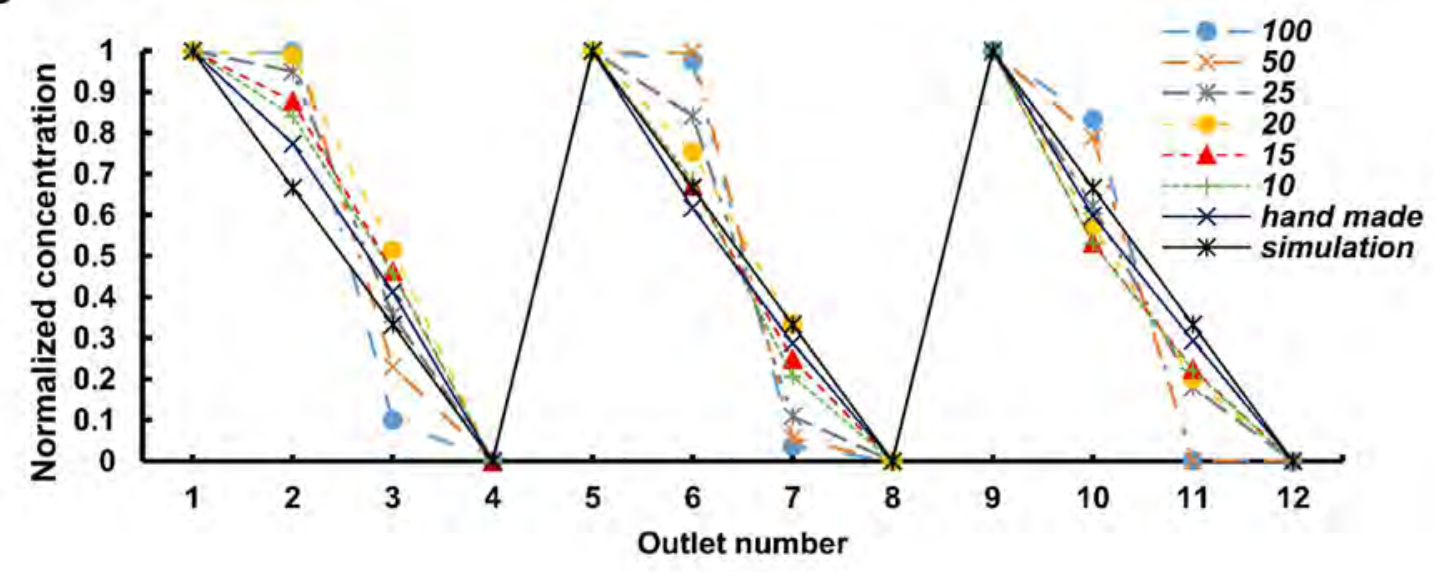

Fig. 8. (A\&B) The first stage and the second stage of the experiment. (C) The output concentration of the device for the flow rate of $20 \mu \mathrm{l} / \mathrm{min}$ using fluorescence emission measurement. The error bars are the range of the three experiments. (The numbers are represented in Fig. 7A) (The unit of the numbers in the legend is $\mu 1 / \mathrm{min}$ ) (D) The output concentration of the device using colorimetric method (The unit of the numbers in the legend is $\mu 1 / \mathrm{min})$. 


\section{Conclusion}

This paper reports a design tool for CGGs. Three different cases were fabricated using the TCDT, and their performance was evaluated experimentally and numerically. TCDT is useful for designing the CGGs made by micromachining, photolithography, and 3D printing. The tool can calculate the dimensions required for making a regular CGG with a number of outlets and non-linear output concentration. In order to validate the model in terms of the fabrication method and the repeatability of the output concentration, the output concentrations were measured using Rhodamine-B and food dye for three different chip designs. The TCDT code can be modified to customize the design of the TCGGs for various lab-on-a-chip applications. The proposed design tool can provide more precise results and a straightforward procedure for CGGs. This tool is placed in the public domain as opensource code. Furthermore, the MDMC method was presented. This method can find the best drug combinations using the multi-drug device, and then using the single-drug device, the best drug concentration of that combination can be found. Based on the fact that non-linear concentration gradients can better investigate the drug concentration changes in dilute solutions, the TCDT can calculate the related calculations and be used in MDMC method.

\section{Conflicts of interest}

The authors declare no conflicts of interest.

\section{References}

Abhyankar, V.V., Lokuta, M.A., Huttenlocher, A., Beebe, D.J., 2006. Characterization of a membrane-based gradient generator for use in cell-signaling studies. Lab on a Chip 6, 389-393. 
Boyden, S., 1962. THE CHEMOTACTIC EFFECT OF MIXTURES OF ANTIBODY AND ANTIGEN ON POLYMORPHONUCLEAR LEUCOCYTES. The Journal of Experimental Medicine 115, 453-466.

Bui, M.-P.N., Li, C.A., Han, K.N., Choo, J., Lee, E.K., Seong, G.H., 2011. Enzyme Kinetic Measurements Using a Droplet-Based Microfluidic System with a Concentration Gradient. Analytical Chemistry 83, 16031608 .

Çengel, Y.A., 2010. Fluid Mechanics. Tata McGraw Hill Education Private.

Chang, C.-W., Cheng, Y.-J., Tu, M., Chen, Y.-H., Peng, C.-C., Liao, W.-H., Tung, Y.-C., 2014a. A polydimethylsiloxane-polycarbonate hybrid microfluidic device capable of generating perpendicular chemical and oxygen gradients for cell culture studies. Lab on a Chip 14, 3762-3772.

Chang, T.C., Mikheev, A.M., Huynh, W., Monnat, R.J., Rostomily, R.C., Folch, A., 2014b. Parallel microfluidic chemosensitivity testing on individual slice cultures. Lab on a Chip 14, 4540-4551.

Chiang, H.-C., Wang, Y.-S., Chou, C.-H., Liao, A.T., Chu, R.-M., Lin, C.-S., 2012. Overexpression of chemokine ligand 7 is associated with the progression of canine transmissible venereal tumor. BMC Veterinary Research 8, 216.

Chung, B.G., Flanagan, L.A., Rhee, S.W., Schwartz, P.H., Lee, A.P., Monuki, E.S., Jeon, N.L., 2005. Human neural stem cell growth and differentiation in a gradient-generating microfluidic device. Lab on a Chip 5, 401406.

Crank, J., 1979. The Mathematics of Diffusion. Clarendon Press.

Diao, J., Young, L., Kim, S., Fogarty, E.A., Heilman, S.M., Zhou, P., Shuler, M.L., Wu, M., DeLisa, M.P., 2006. A three-channel microfluidic device for generating static linear gradients and its application to the quantitative analysis of bacterial chemotaxis. Lab on a Chip 6, 381-388.

DiCicco, M., Neethirajan, S., 2014. An in vitro microfluidic gradient generator platform for antimicrobial testing. BioChip Journal 8, 282-288.

Gao, Y., Sun, J., Lin, W.-H., Webb, D.J., Li, D., 2012. A compact microfluidic gradient generator using passive pumping. Microfluidics and Nanofluidics 12, 887-895.

Gendron, P.-O., Avaltroni, F., Wilkinson, K.J., 2008. Diffusion Coefficients of Several Rhodamine Derivatives as Determined by Pulsed Field Gradient-Nuclear Magnetic Resonance and Fluorescence Correlation Spectroscopy. Journal of Fluorescence 18, 1093.

Girod, B., Niemann, H., Seidel, H.P., 1999. Vision Modelling and Visualization '99. IOS Press, Incorporated. Glawdel, T., Elbuken, C., Lee, L.E., Ren, C.L., 2009. Microfluidic system with integrated electroosmotic pumps, concentration gradient generator and fish cell line (RTgill-W1) - towards water toxicity testing. Lab on a Chip 9, 3243-3250.

Gundersen, R., Barrett, J., 1979. Neuronal chemotaxis: chick dorsal-root axons turn toward high concentrations of nerve growth factor. Science 206, 1079-1080.

Harrison, H., Lu, X., Patel, S., Thomas, C., Todd, A., Johnson, M., Raval, Y., Tzeng, T.-R., Song, Y., Wang, J., Li, D., Xuan, X., 2015. Electrokinetic preconcentration of particles and cells in microfluidic reservoirs. Analyst 140, 2869-2875.

Heo, Y., Cabrera, L., Bormann, C., Shah, C., Takayama, S., Smith, G., 2010. Dynamic microfunnel culture enhances mouse embryo development and pregnancy rates. Human reproduction 25, 613-622.

Hong, B., Xue, P., Wu, Y., Bao, J., Chuah, Y.J., Kang, Y., 2016. A concentration gradient generator on a paper-based microfluidic chip coupled with cell culture microarray for high-throughput drug screening. Biomedical Microdevices 18, 21.

Hu, C., Liu, J., Chen, H., Nie, F., 2017. Microfluidic Platforms for Gradient Generation and its Applications. Biochem Anal Biochem 6, 2161-1009.1000320.

Huang, C.-H., Hou, H.-S., Lo, K.-Y., Cheng, J.-Y., Sun, Y.-S., 2017. Use microfluidic chips to study the effects of ultraviolet lights on human fibroblasts. Microfluidics and Nanofluidics 21, 79.

integra_support, 2017. Serial dilution using 96/384-channel pipette, facrory user manual, p. 21.

Jain, M., Yeung, A., Nandakumar, K., 2010. Induced charge electro-osmotic concentration gradient generator. Biomicrofluidics 4, 014110.

Jang, Y.-H., Hancock, M.J., Kim, S.B., Selimovic, S., Sim, W.Y., Bae, H., Khademhosseini, A., 2011. An integrated microfluidic device for two-dimensional combinatorial dilution. Lab on a Chip 11, 3277-3286. 
Jeon, N.L., Baskaran, H., Dertinger, S.K., Whitesides, G.M., Van De Water, L., Toner, M., 2002. Neutrophil chemotaxis in linear and complex gradients of interleukin- 8 formed in a microfabricated device. Nature biotechnology 20, 826-830.

Jeon, N.L., Dertinger, S.K., Chiu, D.T., Choi, I.S., Stroock, A.D., Whitesides, G.M., 2000. Generation of solution and surface gradients using microfluidic systems. Langmuir 16, 8311-8316.

Jin, D., Ma, X., Luo, Y., Fang, S., Xie, Z., Li, X., Qi, D., Zhang, F., Kong, J., Li, J., 2016. Application of a microfluidic-based perivascular tumor model for testing drug sensitivity in head and neck cancers and toxicity in endothelium. RSC Advances 6, 29598-29607.

Joanne Wang, C., Li, X., Lin, B., Shim, S., Ming, G.-1., Levchenko, A., 2008. A microfluidics-based turning assay reveals complex growth cone responses to integrated gradients of substrate-bound ECM molecules and diffusible guidance cues. Lab on a Chip 8, 227-237.

Kashaninejad, N., Nikmaneshi, M.R., Moghadas, H., Kiyoumarsi Oskouei, A., Rismanian, M., Barisam, M., Saidi, M.S., Firoozabadi, B., 2016. Organ-Tumor-on-a-Chip for Chemosensitivity Assay: A Critical Review. Micromachines 7, 130.

Keenan, T.M., Folch, A., 2008. Biomolecular gradients in cell culture systems. Lab on a Chip 8, 34-57.

Kendig, S., 2003. Use of Microchannels to Determine Diffusion of Food Coloring in Water. MICROELECTRONICS PROCESSING TECHNOLOGY FALL TERM

Kilinc, D., Schwab, J., Rampini, S., Ikpekha, O.W., Thampi, A., Blasiak, A., Li, P., Schwamborn, R., Kolch, W., Matallanas, D., Lee, G.U., 2016. A microfluidic dual gradient generator for conducting cell-based drug combination assays. Integrative Biology 8, 39-49.

Kothapalli, C.R., van Veen, E., de Valence, S., Chung, S., Zervantonakis, I.K., Gertler, F.B., Kamm, R.D., 2011. A high-throughput microfluidic assay to study neurite response to growth factor gradients. Lab on a Chip 11, 497-507.

Kwapiszewska, K., Żukowski, K., Kwapiszewski, R., Brzózka, Z., 2016. Double casting prototyping with a thermal aging step for fabrication of 3D microstructures in poly (dimethylsiloxane). AIMS Biophysics 3, 553562.

Lee, J.M., Kim, J.-e., Kang, E., Lee, S.-H., Chung, B.G., 2011. An integrated microfluidic culture device to regulate endothelial cell differentiation from embryonic stem cells. ELECTROPHORESIS 32, 3133-3137.

Lee, K., Kim, C., Jung, G., Kim, T.S., Kang, J.Y., Oh, K.W., 2010. Microfluidic network-based combinatorial dilution device for high throughput screening and optimization. Microfluidics and Nanofluidics 8, 677-685. Ligrani, P.M., 1994. A study of Dean vortex development and structure in a curved rectangular channel with aspect ratio of 40 at Dean numbers up to 430.

Lim, W., Park, S., 2018. A Microfluidic Spheroid Culture Device with a Concentration Gradient Generator for High-Throughput Screening of Drug Efficacy. Molecules 23, 3355.

Lin, F., Saadi, W., Rhee, S.W., Wang, S.-J., Mittal, S., Jeon, N.L., 2004. Generation of dynamic temporal and spatial concentration gradients using microfluidic devices. Lab on a Chip 4, 164-167.

Liu, M.C., Ho, D., Tai, Y.-C., 2008. Monolithic fabrication of three-dimensional microfluidic networks for constructing cell culture array with an integrated combinatorial mixer. Sensors and Actuators B: Chemical 129, 826-833.

Liu, W., Ren, Y., Chen, F., Song, J., Tao, Y., Du, K., Wu, Q., 2019. A microscopic physical description of electrothermal-induced flow for control of ion current transport in microfluidics interfacing nanofluidics. ELECTROPHORESIS.

Mark, D., von Stetten, F., Zengerle, R., 2012. Microfluidic Apps for off-the-shelf instruments. Lab on a Chip 12, 2464-2468.

Moshksayan, K., Kashaninejad, N., Saidi, M., 2018a. Inventions and Innovations in Preclinical Platforms for Cancer Research. Inventions 3, 43.

Moshksayan, K., Kashaninejad, N., Warkiani, M.E., Lock, J.G., Moghadas, H., Firoozabadi, B., Saidi, M.S., Nguyen, N.-T., 2018b. Spheroids-on-a-chip: Recent advances and design considerations in microfluidic platforms for spheroid formation and culture. Sensors and Actuators B: Chemical 263, 151-176.

Nguyen, B., Graham, P.J., Sinton, D., 2016. Dual gradients of light intensity and nutrient concentration for full-factorial mapping of photosynthetic productivity. Lab on a Chip 16, 2785-2790. 
Nguyen, N.-T., 2012a. Chapter 2 - Fundamentals of mass transport in the microscale, Micromixers (Second Edition). William Andrew Publishing, Oxford, pp. 9-72.

Nguyen, N.-T., 2012b. Chapter 9 - Application of micromixers, Micromixers (Second Edition). William Andrew Publishing, Oxford, pp. 321-342.

Park, J.Y., Hwang, C.M., Lee, S.H., Lee, S.-H., 2007. Gradient generation by an osmotic pump and the behavior of human mesenchymal stem cells under the fetal bovine serum concentration gradient. Lab on a Chip 7, 1673-1680.

Python, 2019. Software Foundation, Python Language Reference, version 3.7.

Ramesan, S., Rezk, A.R., Cheng, K.W., Chan, P.P., Yeo, L.Y., 2016. Acoustically-driven thread-based tuneable gradient generators. Lab on a Chip 16, 2820-2828.

Rao, S.S., 1996. Engineering optimization. Wiley.

Ren, Y., Song, C., Liu, W., Jiang, T., Song, J., Wu, Q., Jiang, H., 2019. On hybrid electroosmotic kinetics for field-effect-reconfigurable nanoparticle trapping in a four-terminal spiral microelectrode array. ELECTROPHORESIS 40, 979-992.

Rismanian, M., Saidi, M.S., Kashaninejad, N., 2019. A new non-dimensional parameter to obtain the minimum mixing length in tree-like concentration gradient generators. Chemical Engineering Science 195, 120-126.

Ruppen, J., Wildhaber, F.D., Strub, C., Hall, S.R.R., Schmid, R.A., Geiser, T., Guenat, O.T., 2015. Towards personalized medicine: chemosensitivity assays of patient lung cancer cell spheroids in a perfused microfluidic platform. Lab on a Chip 15, 3076-3085.

Shames, I.H., 2003. Mechanics of Fluids. McGraw-Hill.

Toh, A.G., Wang, Z., Yang, C., Nguyen, N.-T., 2014. Engineering microfluidic concentration gradient generators for biological applications. Microfluidics and Nanofluidics 16, 1-18.

Tretkoff, E., 2005. Building a Better Fuel Cell Using Microfluidics. APS news 14.

Truskey, G.A., Yuan, F., Katz, D.F., 2004. Transport phenomena in biological systems.

Walker, G.M., Sai, J., Richmond, A., Stremler, M., Chung, C.Y., Wikswo, J.P., 2005. Effects of flow and diffusion on chemotaxis studies in a microfabricated gradient generator. Lab on a Chip 5, 611-618.

Wang, F., 2009. The signaling mechanisms underlying cell polarity and chemotaxis. Cold Spring Harbor perspectives in biology 1.

Yang, C.-G., Wu, Y.-F., Xu, Z.-R., Wang, J.-H., 2011. A radial microfluidic concentration gradient generator with high-density channels for cell apoptosis assay. Lab on a Chip 11, 3305-3312.

Ye, N., Qin, J., Shi, W., Liu, X., Lin, B., 2007. Cell-based high content screening using an integrated microfluidic device. Lab on a Chip 7, 1696-1704.

Zicha, D., Dunn, G.A., Brown, A.F., 1991. A new direct-viewing chemotaxis chamber. Journal of Cell Science 99, 769-775.

Zigmond SH, H.J., 1973. Leukocyte locomotion and chemotaxis New methods for evaluation and demostration of a celldervice chemotactic factor. J Exp Med 137, 23. 